\title{
A TABLEAU APPROACH OF THE KSS NEST
}

\author{
WENJUAN PENG, WEIYUAN QIU, PASCALE ROESCH, LEI TAN, \\ AND YONGCHENG YIN
}

\begin{abstract}
The KSS nest is a sophisticated choice of puzzle pieces given in [Ann. of Math. 165 (2007), 749-841]. This nest, once combined with the KLLemma, has proven to be a powerful machinery, leading to several important advancements in the field of holomorphic dynamics. We give here a presentation of the KSS nest in terms of tableau. This is an effective language invented by Branner and Hubbard to deal with the complexity of the dynamics of puzzle pieces. We show, in a typical situation, how to make the combination between the KSS nest and the KL-Lemma. One consequence of this is the recently proved Branner-Hubbard conjecture. Our estimates here can be used to give an alternative proof of the rigidity property.
\end{abstract}

\section{INTRODUCTION}

Branner and Hubbard proved the follow statement in the case of cubic polynomials:

Let $G$ be a polynomial, with filled Julia set $K_{G}$. Assume that every connected component of $K_{G}$ containing a critical point is aperiodic under the iteration of $G$; then $K_{G}$ is a Cantor set.

They then conjectured that the statement should be true in its full generality. This conjecture has been recently proved independently by Kozlovski-van Strien and by Qiu-Yin $[\mathrm{KS}, \mathrm{QY}$.

The starting point of the proof is the same as that of [BH]: it consists of building a puzzle dynamical system surrounding $K_{G}$ and of controlling the moduli of annuli lying between various puzzle pieces.

The theory of puzzle dynamics has since been greatly developed. Among many of the new techniques, the combination of the KSS nest and the KL-Lemma has shown

Received by the editors March 6, 2009.

2010 Mathematics Subject Classification. Primary 32H50, 37F10, 37F20.

The first author is supported by China Postdoctoral Science Foundation under Grant No. 20080440270, National Natural Science Foundation of China under Grant No. 10831004 and the Doctoral Education Program Foundation of China under Grant No. 20060001003.

The second author is supported by National Natural Science Foundation of China under Grants No. 10831004 and 10871047.

The third author is supported by EU Research Training Network CODY, Conformal Structures and Dynamics.

The fourth author is supported by National Natural Science Foundation of China under Grant No. 10831004.

The fifth author is supported by the project ABC of the Agence Nationale de la Recherche Francaise.

(C)2010 American Mathematical Society Reverts to public domain 28 years from publication 
to be a very powerful machinery, and has led to several important advancements in the field, including the proof of the above conjecture.

Roughly speaking, this new machinery solves the two main problems that appear when one wants to generalize $[\mathrm{BH}$, that is how to "estimate" moduli of annuli when one allows higher degree critical points and also how to control the mutual recurrence among various critical points.

In this note we will illustrate the power of this machinery in a typical situation, by proving Theorem 2.1 below. It is the key new ingredient in proving the Branner Hubbard conjecture (see [KS, QY]).

The presentation here is however entirely different from the existing literature. At first we single out the combinatorial part (control of degree of puzzle dynamics) into one intermediate statement (Proposition [3.2), and in the space of one page, we prove the analytic part (i.e. application of the Kahn-Lyubich covering lemma for the control of moduli). We then express the complexity of puzzle dynamics exclusively in the tableau language of Branner-Hubbard, thus reducing their study into the study of a combinatorial dynamical system on the tableau. Finally, we provide three variants for the proof of Proposition 3.2 one for a shortcutted KSS nest, one for the original KSS nest, and one for the unicritical setting. Comparing the three will give a fairly good understanding of the underlying mechanism.

We give some sharpened estimates, reducing for instance the operating time of the last successor operator from $3 b$ (in QY]) or $5 b$ (in [KSS, [KS] to $b+$ 2 (where $b$ is the number of critical points). We use these to show that our short-cutted KSS nest is already sufficient to prove Theorem 2.1. This simplifies the existing proofs, and at the same time unifies the KSS nest in the multicritical setting with that in the unicritical setting (see Appendix D, where we also include a variant of Branner-Hubbard's original construction for the sake of comparison).

Our estimates here are the starting point to give an alternative proof of the rigidity property, following the method of AKLS in the unicritical setting; see $[\mathrm{PT}$.

Our presentation will rely only on the (highly non-trivial) Kahn-Lyubich covering lemma, together with a few basic tableau rules and moduli inequalities. Everything else will be self-contained. In particular, we include the complete proof of two technical results: the existence of at least two successors and the construction of annuli that avoid the post-critical set. They are absolutely fundamental but their proofs are difficult to find in the literature.

We introduce the concept of upper triangles and parallelograms in the tableau. This turns out to be an effective alternative to the indice numerations of tableau entries.

The scheme of the article is the following. Section 2 gives the Setup, definitions and results. In section 3 we explain how to apply the Kahn-Lyubich Lemma assuming the existence of the KSS nest. Section 4 describes the construction of the KSS nest, as well as their properties, pushing two technical results in the appendices for the clarity of the exposition. The appendices also contain a table of various constants.

For historical comments of this machinery, please refer to [KSS, KS, QY]. 


\section{Statement}

The Setup.

$\mathbf{V}=\bigsqcup_{i \in I} V_{i}$ is the disjoint union of finitely many simply connected hyperbolic Riemann surfaces,

$\mathbf{U}$ is compactly contained in $\mathbf{V}$,

and is the union of finitely many hyperbolic discs with disjoint closures;

$f: \mathbf{U} \rightarrow \mathbf{V}$ is a proper holomorphic map

with all critical points contained in

$$
K_{f}:=\left\{z \in \mathbf{U}, f^{n}(z) \in \mathbf{U} \forall n\right\}
$$

with each $\mathbf{V}$-component containing at most one critical point.

Since we are mainly interested in the properties of $K_{f}$, reducing $\mathbf{V}$ if necessary, we may and will always assume that each $\mathbf{V}$-component is a Jordan disc contained in some Riemann surface. This implies that each $\mathbf{U}$-component is a Jordan disc contained in a $\mathbf{V}$-component.

The connected components of $f^{-m}(\mathbf{V})$ are called puzzle pieces of depth $m$.

For any $x \in K_{f}$, the tableau $\mathcal{T}(x)$, following Branner-Hubbard [BH], is the graph embedded in $\left\{(u, v), u \in \mathbb{R}^{-}, v \in \mathbb{R}\right\}$ with the axis of $u$ pointing upwards and the axis of $v$ pointing rightwards (this is the standard $\mathbb{R}^{2}$ with reversed orientation), with vertices indexed by $-\mathbb{N} \times \mathbb{N}$, where $\mathbb{N}=\{0,1, \cdots\}$, with the vertex at $(-m, 0)$ being $P_{m}(x)$, the puzzle piece of depth $m$ containing $x$, and with $f^{j}\left(P_{m}(x)\right)$ occupying the $(-m+j, j)$ th entry of $\mathcal{T}(x)$. Therefore a given puzzle piece $Q$ may appear at different entries of $\mathcal{T}(x)$ for different $x$ 's, but will always be on the same row, denoted by $\operatorname{row}(Q)$. There are three types of edges: vertical, horizontal and diagonal. It is also equipped with the graph metric so that each edge is isometric to the unit interval $[0,1]$.

For a vertex $Q$ with index $(-m, n)$, we say that $m$ is the depth, or the row number of $Q$.

A vertical segment, say bounded by two vertices $E$ and $F$, is assigned a length, denoted by $|\underset{F}{E}|$, which is simply the depth difference between $F$ and $E$. It is also assigned a modulus, equal to $\bmod (E \backslash \bar{F})$. Recall that modulus of an annulus $A$ is a conformal invariant, and is defined to be $\frac{1}{2 \pi} \log R$ if $A$ is mapped conformally onto $\{1<|z|<R\}$ (see e.g. [M2]).

The map $f$ induces a (partial) dynamical system, indicated by the diagonal edges, mapping $\mathcal{T}(x) \backslash\{0-$ th row $\}$ onto $\mathcal{T}(x) \backslash\{0-$ th column $\}=\mathcal{T}(f(x))$; see Figure 1 .

For two puzzle pieces $Q$ and $I$ such that $f^{k}(Q)=I$ for some $k>0$, we use $\left.{ }_{[Q} /{ }^{I}\right]$ to denote the diagonal segment from $Q$ to $I$. Thus the consecutive vertices on this segment are $Q, f(Q), f^{2}(Q), \cdots, f^{k}(Q)=I$. We use also $\left[Q /{ }^{I[}\right.$ if we exclude $I$. Define $]_{Q} /^{I]}$ and $]_{Q} /^{I[}$ accordingly. This diagonal segment (whether it is closed, half open or open) is assigned a length, denoted by $\left.\right|_{Q} /{ }^{I} \mid$, which is simply the depth difference between $Q$ and $I$. It is also assigned a degree, equal to $\operatorname{deg}\left(f^{k}: Q \rightarrow I\right)$.

For $c$ a critical point, the nest $\left(P_{m}(c)\right)_{m}$ is called a critical nest.

We associate each critical point with a different color (not white nor black), and color each critical puzzle piece accordingly. 


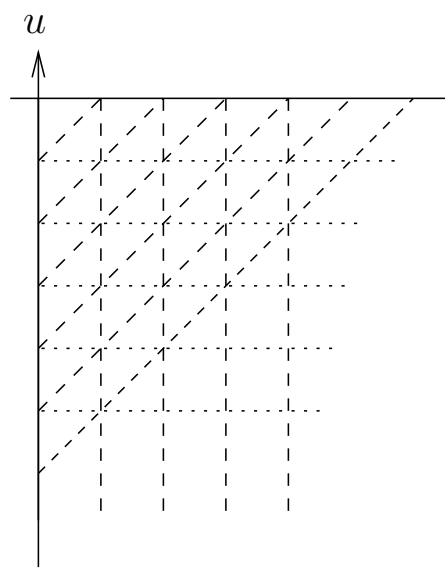

$\mathcal{T}(x) \subset \mathbb{R}_{u}^{-} \times \mathbb{R}_{v}^{+}$a graph with

- $\{$ vertices $\}=-\mathbb{N} \times \mathbb{N}$, some are colored

- 3 types of edges: -, |, / (each of length 1 )

- a modulus $\mu(a)>0$ for each vertical segment $a$ with $\mu(a \cup b) \geq \mu(a)+\mu(b)$ if $a, b$ are consecutive

- a degree $\operatorname{deg}(a) \geq 1$ for each diagonal segment $a$ with $\operatorname{deg}(a \cup b)=\operatorname{deg}(a) \operatorname{deg}(b)$ if $a, b$ are consecutive

$\operatorname{deg}(/$ edge $)>1$ iff the left vertex is colored

Figure 1. Tableau

A vertex in a tableau is sometimes marked by $\circ$ if it is non-critical, by a solid colored $\bullet$ if it is a critical puzzle piece of the corresponding color, and by $\times$ if it is unknown. Thus tableau entries with identical depth and color (but not white nor black) represent the same critical puzzle piece.

A successor of a critical puzzle piece $P$ is a critical puzzle piece $S$ that is both a sub-piece of $P$ and a pullback of $P$, such that ${ }_{[S} /^{P]}$ meets every critical color at most twice; see Figure 2 .

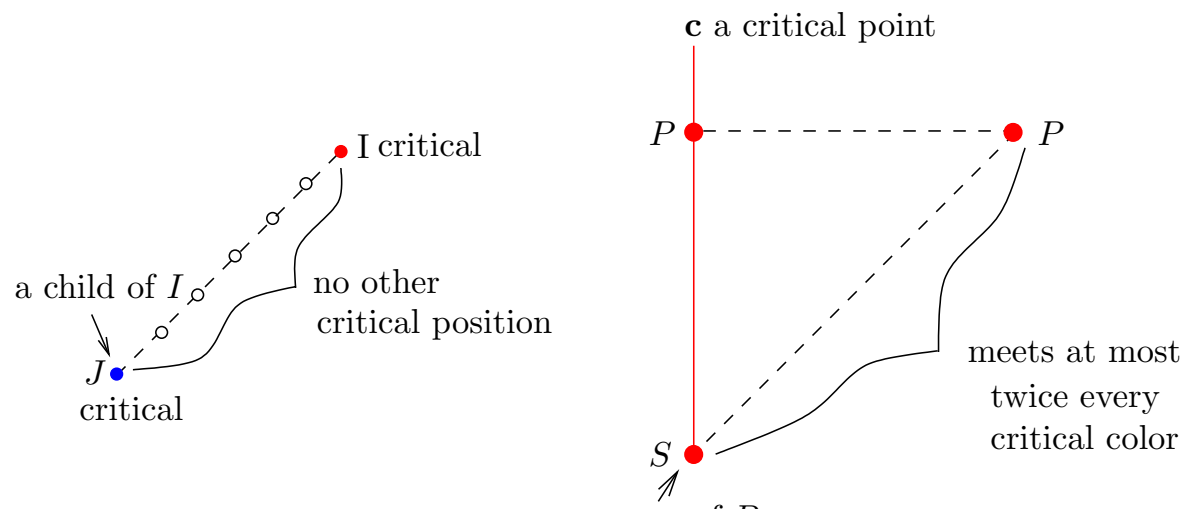

a successor of $P$

Figure 2. Child and successor

Hypothesis of recurrence. Let $f$ be as in the setup. Denote by Crit $(f)$ the set of critical points of $f$. In this paper we assume:

- given any couple $\left(c, c^{\prime}\right) \in C r i t(f)^{2}$, marching horizontally to the right of any vertex in $\mathcal{T}(c)$, one will meet a vertex that has the color of $c^{\prime}$; and $\mathcal{T}(c)$ does not 
contain a full column whose vertices have the color of $c$ (except of course on the 0-th column);

- any critical piece of any color has at most finitely many successors.

(We will prove in Appendix C that this is equivalent to the commonly called persistently recurrent condition, i.e. with 'successors' replaced by the notion children, whose definition will be given in Appendix C.)

Set $\widehat{\mathcal{P}}_{f}=\bigcup_{n \geq 0} \bigcup_{c \in C r i t(f)}\left\{f^{n}(c)\right\}$. Note that we include the critical points in $\widehat{\mathcal{P}}_{f}$. Set

$$
b=\# \text { Crit }(f) \quad \text { and } \quad \delta:=\sup _{c \in C r i t(f)} \operatorname{deg}_{c}(f)
$$

(these numbers are necessarily finite). Set also

$\widehat{\mu}=\min \left\{\bmod \left(P_{0}(c) \backslash \bar{W}\right) \mid c \in C r i t(f), W\right.$ a component of $\mathbf{U}$ contained in $\left.P_{0}(c)\right\}$.

The objective of this note is to present a proof of the following:

Theorem 2.1. Let $f$ be a map satisfying the hypothesis of recurrence. There is a constant $C(b, \delta, \widehat{\mu})>0$, depending only on $b, \delta$ and $\widehat{\mu}$, such that for any $c \in C r i t(f)$, there is a sequence of puzzle pieces $P_{m_{n}}(c)$, with $m_{n} \nearrow+\infty$ (exponentially fast), such that for each $n$, the annulus $P_{m_{n-1}}(c) \backslash \overline{P_{m_{n}}(c)}$ contains an essential annulus $A_{n}$ whose modulus is bounded from below by $C(b, \delta, \widehat{\mu})$. Moreover $A_{n} \cap \widehat{\mathcal{P}}_{f}=\emptyset$.

Applying then Grötzsch's inequality one could conclude immediately that the diameter of $P_{m}(c)$ tends to 0 as $m \rightarrow \infty$ and the connected component of $K_{f}$ containing $c$ is reduced to the single point set $\{c\}$. To see how to deduce the Branner-Hubbard conjecture from this, please refer to [KS, QY].

For the unicritical (i.e. $b=1$ ) version of this theorem, see [KL2, TY and Appendix D

Sketch of the proof. Given $c \in C r i t(f)$, starting from $K_{0}=P_{0}(c)$, and for any $n \geq 1$, we define inductively a double sequence of puzzle pieces $\left(K_{n}^{\prime}, K_{n}\right)$ satisfying $K_{n-1} \ni K_{n}^{\prime} \ni K_{n} \ni c$ following Kozlovski-Shen-Strien. We then prove the theorem for $P_{m_{n}}(c)=K_{n}$ and $A_{n}=K_{n}^{\prime} \backslash \bar{K}_{n}$, using the specific combinatorics and the Kahn-Lyubich covering lemma.

\section{Reduction to A CONTRol of DEGReE}

Lemma 3.1 (Kahn-Lyubich Covering Lemma). Fix $\eta>0$ and $D \in \mathbb{N}$. There is $\varepsilon(\eta, D)>0$ such that: given any $g: U \rightarrow V$,

$$
\begin{aligned}
& A \Subset A^{\prime} \Subset U, B \Subset B^{\prime} \Subset V \text { (all discs) }, \\
& g: U \rightarrow V, A^{\prime} \rightarrow B^{\prime}, A \rightarrow B \text { are proper holomorphic maps, } \\
& \left\{\begin{array}{l}
\operatorname{deg}\left(\left.g\right|_{U}\right) \leq D \\
\operatorname{deg}\left(\left.g\right|_{A^{\prime}}\right) \leq d \\
\bmod \left(B^{\prime} \backslash \bar{B}\right) \geq \eta \cdot \bmod (U \backslash \bar{A})
\end{array} \Longrightarrow \bmod (U \backslash \bar{A}) \stackrel{\text { either }}{>} \stackrel{\stackrel{\text { or }}{>}}{>} \frac{\eta}{2 d^{2}} \bmod (V \backslash \bar{B}) .\right.
\end{aligned}
$$




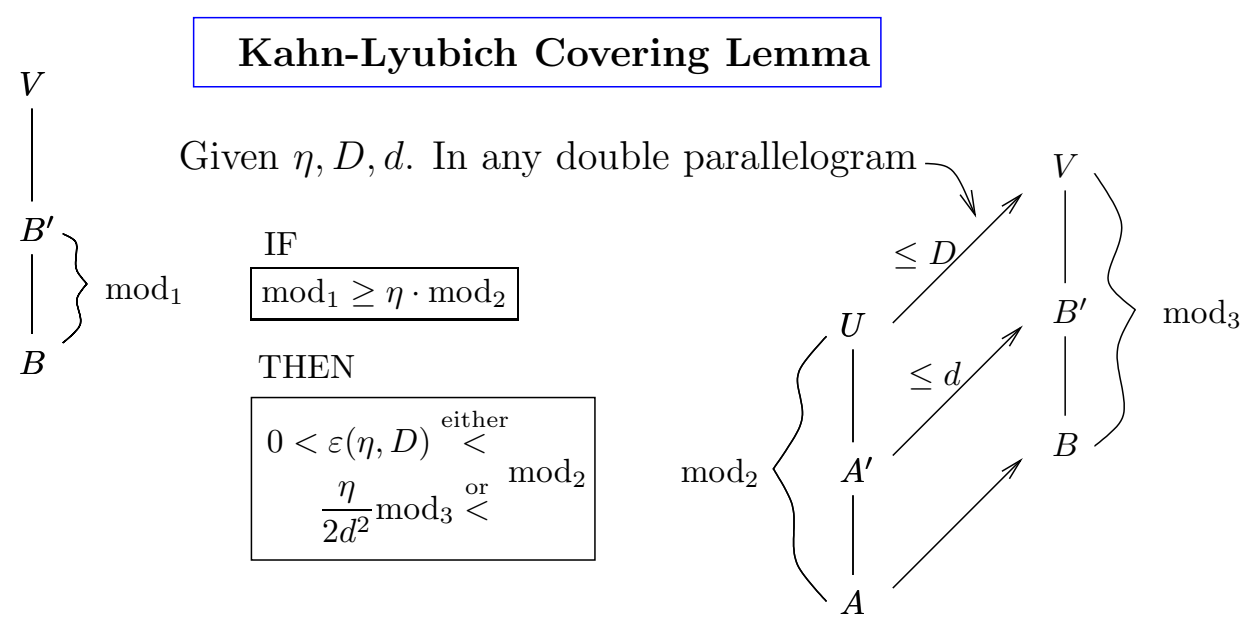

Let $K_{0}$ be a critical puzzle piece, say containing the critical point c. Assume that $\left(K_{n}^{\prime}, K_{n}\right)_{n \geq 1}$ is a sequence of pairs of critical puzzle pieces satisfying (see Figure 3):

$$
\left\{\begin{array}{l}
K_{0} \ni K_{1}^{\prime} \ni K_{1} \ni K_{2}^{\prime} \ni K_{2} \ni \cdots ; \\
\forall n \geq 1, \quad \text { both } K_{n}^{\prime} \text { and } K_{n} \text { are pullbacks of } K_{n-1} ; \\
\forall n \geq 1, \quad\left(K_{n}^{\prime} \backslash \bar{K}_{n}\right) \cap \widehat{\mathcal{P}}_{f}=\emptyset .
\end{array}\right.
$$

For any $m \geq 1$, we construct a KL-map $g:\left(U, A^{\prime}, A\right) \rightarrow\left(V, B^{\prime}, B\right)$ as follows (see Figure 3). We will look at $\mathcal{T}(\mathbf{c})$, rows of $K_{0}, K_{m}, K_{m+2}^{\prime}$ and $K_{m+2}$.

Set $\xi=||_{K_{m+2}^{\prime}} /^{K_{m}}|, M=|_{K_{m}} /^{K_{0}} \mid, \hat{x}=f^{\xi}(\mathbf{c})$ and $\hat{y}=f^{M}(\hat{x})$. Set the triple $U, V, g$ as:

$$
\begin{array}{cc}
V:=K_{0} & -\quad V \\
U:=K_{m} & \nearrow_{g:=f^{M}}
\end{array}
$$

By the hypothesis of recurrence, there is a minimal $l \geq 0$ such that from the $\mathcal{T}(\mathbf{c})$ column of $\hat{y}$, row $\left(K_{m}\right)$, marching horizontally to the right $l$ steps, one will again meet $K_{m}$. If $l=0$, i.e. $\hat{y} \in K_{m}$, set $B=K_{m}$ and $B^{\prime}=K_{m}^{\prime}$. If $l>0$, let $B^{\prime} \ni B$ be the pair of puzzle pieces on $\operatorname{column}(\hat{y})$ forming a parallelogram with $K_{m}^{\prime}, K_{m}$ (i.e. $\hat{y} \in B$ and $\left.f^{l}(B)=K_{m}\right)$. In both cases let $A^{\prime} \ni A$ be the pair of puzzle pieces on $\operatorname{column}(\hat{x})$ forming a parallelogram with $B^{\prime}, B$.

Proposition 3.2. Starting from any critical piece $K_{0}$, there is a sequence of pairs of critical pieces $\left(K_{n}^{\prime}, K_{n}\right)_{n \geq 1}$ satisfying (21), such that, for any $m \geq 1$, for the maps $g, f^{\xi}$ constructed above, there are integers $C_{K}, d, \beta$ depending only on $b, \delta$, and there is $\alpha=\delta^{b-1}$, such that:

(a) $\operatorname{deg}\left(K_{m} / K^{K_{m-1}}\right) \leq C_{K}, \quad \operatorname{deg}\left(K_{m}^{\prime} / K_{m-1}^{K_{m-1}}\right) \leq C_{K}$,

(b) $\operatorname{deg}\left(B^{\prime} / K_{m}^{\prime}\right) \leq \alpha$,

(c) $\operatorname{deg}\left(A^{\prime} / B^{\prime}\right) \leq d$,

(d) $\operatorname{deg}\left(K_{m+2}^{\prime} /^{U}\right)=\operatorname{deg}\left(K_{m+2}^{\prime} / K_{m}^{K_{m}}\right) \leq \beta$,

(e) $f^{\xi}\left(K_{m+2}\right) \subset A$.

Moreover, the sequences $\operatorname{depth}\left(K_{n}\right)$, depth $\left(K_{n}\right)-\operatorname{depth}\left(K_{n-1}\right)$ and $\operatorname{depth}\left(K_{n}\right)-$ $\operatorname{depth}\left(K_{n}^{\prime}\right)$ all tend to $+\infty$ exponentially. 
Proposition 3.2

$\exists\left(K_{n}^{\prime}, K_{n}\right)_{n}$ such that

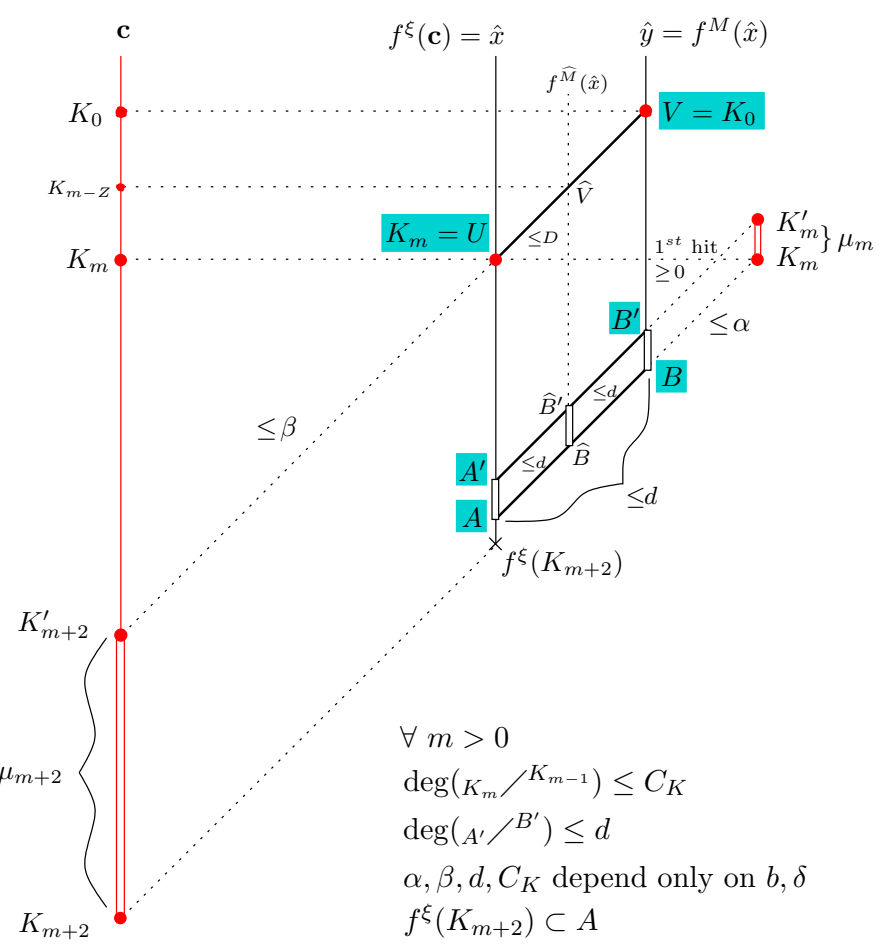

Figure 3. Construction of KL-maps from a KSS nest

Assuming this, we will give the following.

Proof of Theorem 2.1. We will prove:

Claim. Fix any $c \in \operatorname{Crit}(f)$ and set $K_{0}=P_{0}(c)$. Then for any double sequence $\left(K_{n}^{\prime}, K_{n}\right)_{n>1}$ satisfying the properties in Proposition $3.2 \bmod \left(K_{n}^{\prime} \backslash \bar{K}_{n}\right) \geq C$ for some $C>0$ depending only on $b, \delta, \widehat{\mu}$.

Set $Z=2 d^{3} \alpha^{2} \beta^{2}+1$. It depends only on $b, \delta$. Set $\mu_{n}=\bmod \left(K_{n}^{\prime} \backslash \bar{K}_{n}\right), n \geq 1$.

Case 1. Assume there is an increasing sequence $k_{n} \rightarrow \infty$ such that $\mu_{m^{\prime}} \stackrel{\forall m^{\prime}<k_{n}}{\geq} \mu_{k_{n}}$.

There is $n_{0}$ so that any $n \geq n_{0}$ satisfies $k_{n}-2 \geq Z$.

Fix $n \geq n_{0}$ and set $m=k_{n}-2$. Then $\mu_{m^{\prime}} \geq \mu_{m+2}$ for any $m^{\prime} \leq m+2$. For this $m$, define, as above, the collection of objects

$$
\left(\xi, M, U, V, g, B, B^{\prime}, A, A^{\prime}\right)
$$

We do not apply directly the Kahn-Lyubich covering lemma to the map $g$, as the degree of $g$ depends on $m$ (therefore on $n$ ). Instead we will deduce a KL-map $\widehat{g}$ with degree independent of $m$ (therefore of $n$ ), by setting:

$$
\begin{aligned}
& \widehat{V}=K_{m-Z} ; \\
& \widehat{g}=f^{\widehat{M}}: U \rightarrow \widehat{V} \text { where } \widehat{M} \text { is chosen so that } f^{\widehat{M}}(U)=\widehat{V} ; \\
& \widehat{B}^{\prime}=\widehat{g}\left(A^{\prime}\right) \text { and } \widehat{B}=\widehat{g}(A) .
\end{aligned}
$$


Note that, by (a) above, $\operatorname{deg}(\widehat{g}) \leq C_{K}^{Z}=: D$ with $D$ independent of $m$. Note also

$$
\begin{gathered}
\operatorname{deg}\left(A^{\prime} /^{\widehat{B}^{\prime}}\right), \operatorname{deg}\left(\widehat{B}^{\prime} /{ }^{B^{\prime}}\right) \leq \operatorname{deg}\left(A^{\prime} / B^{B^{\prime}}\right) \stackrel{(c)}{\leq} d, \\
\operatorname{deg}\left(\widehat{B}^{\prime} / K_{m}^{\prime}\right)=\operatorname{deg}\left(\widehat{B}^{\prime} /^{B^{\prime}}\right) \operatorname{deg}\left(B^{\prime} / K_{m}^{\prime}\right) \stackrel{(b)}{\leq} d \alpha .
\end{gathered}
$$

We have (see Figure 33),

$$
\mu_{m+2} \geq \frac{\left.\bmod \left(U \backslash \overline{f^{\xi}\left(K_{m+2}\right.}\right)\right)}{\operatorname{deg}\left(K_{m+2}^{\prime} / U\right)} \underset{(\bar{d})}{\geq} \frac{\bmod (U \backslash \bar{A})}{\beta} ;
$$

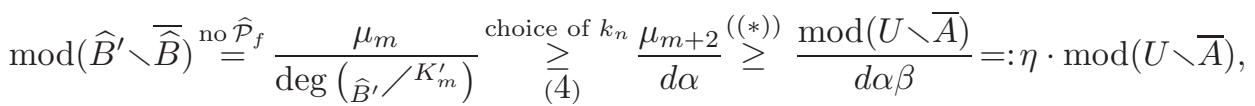

with $\eta:=\frac{1}{d \alpha \beta}$ independent of $m$.

Therefore for any $n \geq n_{0}$ and $m=k_{n}-2$, we may then apply Kahn-Lyubich Lemma to $\widehat{g}:\left(U, A^{\prime}, A\right) \rightarrow\left(\widehat{V}, \widehat{B}^{\prime}, \widehat{B}\right)$, to conclude that,

$$
\begin{aligned}
\beta \cdot \mu_{m+2} \stackrel{[*)}{\geq} \bmod (U \backslash \bar{A}) \stackrel{\text { either }}{>} \stackrel{\stackrel{\text { or }}{>}}{>} \frac{\eta \cdot \bmod (\widehat{V} \backslash \widehat{\widehat{B}})}{2 \operatorname{deg}\left(A^{\prime} / \widehat{B}^{\prime}\right)^{2}} \stackrel{(*)}{\geq} \frac{\eta}{2 d^{2}}\left(\frac{\mu_{m}}{\alpha}+\cdots+\frac{\mu_{m-Z+1}}{\alpha}\right) \stackrel{\text { choice of } k_{n}}{\geq} \frac{\eta Z}{2 d^{2} \alpha} \mu_{m+2},
\end{aligned}
$$

where the inequality $\left({ }^{*}\right)$ is proved as follows: For $j=m, m-1, \cdots, m-Z+1$, and for $l_{j} \geq 0$ minimal so that $f^{l_{j}}(\widehat{g}(\hat{x})) \in K_{j}$, denote by $B_{j}$, respectively $B_{j}^{\prime}$, the connected component of $f^{-l_{j}}\left(K_{j}\right)$, respectively $f^{-l_{j}}\left(K_{j}^{\prime}\right)$, containing $\widehat{g}(\hat{x})$. Then $\left\{B_{j}^{\prime} \backslash \overline{B_{j}}\right\}_{j}$ are pairwise disjoint essential annuli in $\widehat{V} \backslash \overline{\widehat{B}}$ with $\bmod \left(B_{j}^{\prime} \backslash \overline{B_{j}}\right)=\frac{\mu_{j}}{\operatorname{deg}\left(B_{j} \rightarrow K_{j}\right)} \geq \frac{\mu_{j}}{\alpha}$ (due to $\left(K_{j}^{\prime} \backslash \bar{K}_{j}\right) \cap \widehat{\mathcal{P}}_{f}=\emptyset$ and Formula (5) below, where $\widehat{\mathcal{L}}_{\widehat{g}(\hat{x})}\left(K_{j}\right)=B_{j}$ ).

By our choice of $Z$ we have $Z>2 d^{3} \alpha^{2} \beta^{2}=\frac{2 d^{2} \alpha \beta}{\eta}$. Hence the second line above is impossible. So $\mu_{k_{n}}=\mu_{m+2}>\frac{1}{\beta} \cdot \varepsilon(\eta, D)>0$. Therefore $\forall l \in \mathbb{N}$, $\mu_{l} \geq \lim _{n \rightarrow \infty} \mu_{k_{n}} \geq \frac{1}{\beta} \cdot \varepsilon(\eta, D)$.

Case 2. Assume there is $k_{0}$ such that $\mu_{k} \geq \mu_{k_{0}}$ for all $k \geq 1$.

Case 2.1. $k_{0}-2 \geq Z$. Then repeating the same argument as above we know that $\mu_{k_{0}} \geq \frac{1}{\beta} \cdot \varepsilon(\eta, D)$.

Case 2.2. $k_{0}-2<Z$. Then $k_{0} \leq Z+1$ with $Z+1$ depending only on $b, \delta$. Notice that $K_{k_{0}}^{\prime}$ is mapped by some iterate of $f$ onto $K_{0}=P_{0}(c)$ with degree bounded by $C_{K}^{Z+1}$, due to (a) of Proposition 3.2 The same iterate of $f$ maps $K_{k_{0}}$ into a component $W$ of $\mathbf{U}$ contained in $P_{0}(c)$. So $\mu_{k_{0}} \geq \frac{1}{C_{K}^{Z+1}} \bmod \left(P_{0}(c) \backslash \bar{W}\right) \geq \frac{1}{C_{K}^{Z+1}} \widehat{\mu}$.

We have now proved the theorem for $C=\min \left\{\frac{1}{\beta} \cdot \varepsilon(\eta, D), \frac{1}{C_{K}^{Z+1}} \widehat{\mu}\right\}$.

\section{Construction of the $\operatorname{KSS}$ nest $\left(K_{n}^{\prime}, K_{n}\right)_{n}$}

Following $\mathrm{KSS}$ ] we will define three operators $\Gamma, \mathcal{A}, \mathcal{B}$ from a critical nest into itself. They are return domain operators in the sense that they map a critical piece $I$ to a critical piece $J$ that is both a sub-piece of $I$ and a pullback of $I$. We will then combine them to get the desired double sequence. 
4.1. Tableau rules. To start with, we give the three basic tableau rules of Branner-Hubbard (see [BH] M1] for slightly different conventions, and [Ro] for identical convention as here; see also Figure 4):

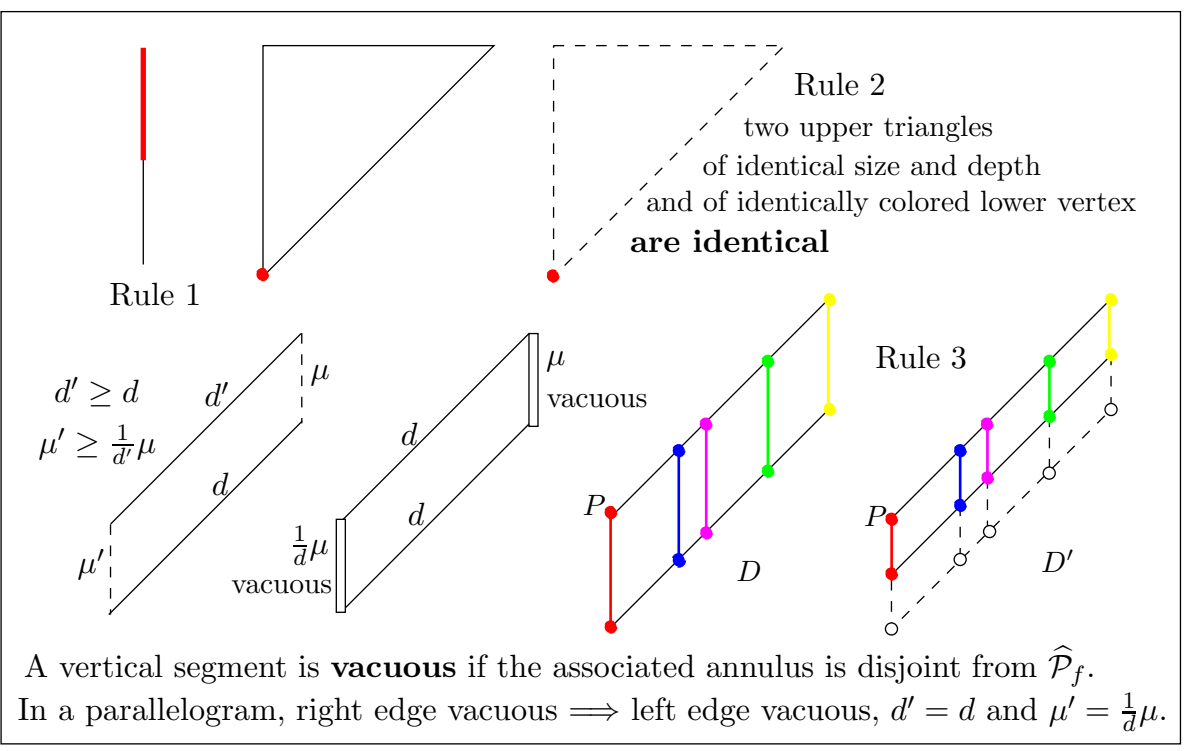

Figure 4. Tableau rules, upper triangles and parallelograms, with $D$ critically full

Rule 1 (vertical segment rule):

In each column either there is no critical vertex or the critical vertices form a single vertical segment of the same color on the top of the column.

An upper triangle $T$ in a tableau is by the definition a sides-included filled triangle bounded by a vertical segment on the left, a horizontal segment on the top and a diagonal segment as the third edge. Its size, denoted by $|T|$, is simply the length of any of its edges, and its depth is the depth of its lowest vertex; see Figure 4.

Rule 2 (double triangle rule):

Two upper triangles in $\mathcal{T}(x)$ and $\mathcal{T}\left(x^{\prime}\right)$ with identical size and lower vertex are identical.

A vertical parallelogram in a tableau is a sides-included filled parallelogram bounded by two vertical segments and two diagonal segments; see Figure 4

A vertical parallelogram $D$ is said to be critically full if its two vertical edges are entirely critical (possibly with different colors), and if every vertical critical segment that touches the top diagonal edge of $D$ (if any) remains critical at least until it reaches the lower diagonal edge of $D$; see Figure 4. 
Rule 3a (double parallelogram rule):

Let $D \subset \mathcal{T}(x), D^{\prime} \subset \mathcal{T}\left(x^{\prime}\right)$ be two vertical parallelograms

with identical size and upper left vertex, such that $D$ is critically full.

Then $D^{\prime}$ is either identical to $D$,

or has its lower diagonal edge completely noncritical.

A vertical segment $\stackrel{J}{I}_{J}^{I}$ is called vacuous if $(I \backslash \bar{J}) \cap \widehat{\mathcal{P}}_{f}=\emptyset$. In other words, in any column of any critical tableau $\mathcal{T}(c)$, whenever $I$ appears, the full vertical segment from $I$ to $J$ appears (otherwise the orbit of $c$ would visit $I \backslash \bar{J}$ ).

Rule 3b:
A vertical parallelogram with right edge vacuous has all
of its vertical segments vacuous, and furthermore,
is critically full, if its two top vertices are both critical.

See Figure 4. This rule can be deduced from the following observation: Let $G: U \rightarrow V$ be a branched covering of two topological discs. Assume that $V$ is further decomposed into $A \sqcup K$ such that $A$ is an open annulus without critical values of $G$ and that $K$ is compact and connected. Then $U=G^{-1}(A) \sqcup G^{-1}(K)$, and $G^{-1}(A)$ is an open annulus whereas $G^{-1}(K)$ is compact, connected and containing all critical points of $G$.

\subsection{First hits have bounded degree.}

$\begin{array}{ccc}\frac{x}{\times} & \frac{x}{1^{\text {st }} \text { hit }} I \text { (critical) } & \times{ }^{1^{\text {st }} \text { hit }} I \text { (critical) } \\ \mid \mathcal{L}_{x}(I) & \widehat{\mathcal{L}}_{x}(I)\end{array}$

For any pair $(I, x)$ such that $I$ is a critical puzzle piece (say colored red), and $x \in K_{f}$, we produce a puzzle piece $\mathcal{L}_{x}(I)$ (called the pullback of the first $\geq 1$ hit of $x$ to $I$ ) as follows: start from the 0 -th column of $\mathcal{T}(x)$ at row $(I)$, march right $k \geq 1$ steps until the first hit of a red spot 1 (if any, this exists always if $x \in \widehat{\mathcal{P}}_{f}$ ). Then that spot represents $I$ and $\mathcal{L}_{x}(I)$ is the pulled-back piece by $f^{k}$ of $I$ containing $x$. It is the lower vertex of an upper triangle whose left edge is on the 0-th column of $\mathcal{T}(x)$, whose right vertex is $I$ and whose top edge does not contain red spots (except at the ends).

Similarly we define the pullback of the first $\geq 0$ hit of $x$ to $I$ to be a puzzle piece $\widehat{\mathcal{L}}_{x}(I)$ containing $x$ which is equal to $I$ if $x \in I$, otherwise is equal to $\mathcal{L}_{x}(I)$.

Lemma 4.1. For $I$ a critical piece and $x \in K_{f}$, each of the half open diagonals $\left[\mathcal{L}_{x}(I){ }^{I[} \text { and }\right]_{\mathcal{L}_{x}(I)}{ }^{I]}$ meets every critical color at most once. And

$$
\operatorname{deg}\left(\mathcal{L}_{x}(I) /^{I}\right) \leq \delta^{b} ; \quad \operatorname{deg}\left(\widehat{\mathcal{L}}_{x}(I) /^{I}\right) \leq \delta^{b-1}=: \alpha .
$$

Proof. Otherwise we get two upper triangles of different sizes, both with $I$ as the right vertex, and both have a vertical left edge entirely critical of the same coloring. Say $I$ is red. Now moving the smaller triangle to the left and applying Rule 2 would imply an earlier hit of red spots on the top edge of the larger triangle.

\footnotetext{
${ }^{1}$ We will frequently use the word 'spot' for a vertex in a tableau.
} 
4.3. Last successor operator $\Gamma$. In this subsection we fix $\mathbf{c} \in C r i t(f)$, say colored red, and we fix $J$ any choice of a puzzle piece containing $\mathbf{c}$.

Lemma 4.2 (a consequence of the hypothesis of recurrence).

(a) $2 \leq \#\{$ successors of $J\}<+\infty$.

Denote by $\Gamma(J)$ the last successor of $J$, i.e. the successor with the greatest depth. Then

$$
\text { (b) }\left\{\begin{array}{l}
\operatorname{deg}(\Gamma(J) / J) \leq \delta^{2 b-1}=: C_{\Gamma} \\
\#(\{\text { red spots }\} \cap[\Gamma(J) / J[)=1 .
\end{array}\right.
$$

We will postpone the proof of this lemma to Appendix A. The main difficulty lies in the part where there are at least two successors.

Definition. Denote by $r(J)$ (respectively $R(J)$ ) the minimal (respectively maximal) length of a horizontal segment linking two consecutive $J$ vertices, in all $\mathcal{T}\left(c^{\prime}\right), c^{\prime} \in$ Crit $(f)$.

We might have $R(J)=+\infty$ a priori, but the following corollary of Lemma 4.2 will exclude this possibility. The estimates here play an essential role in the sequel.

Corollary 4.3. $\quad$ (i) If $S$ is a critical sub-piece of $J$, then $R(S) \geq R(J)$ and $r(S) \geq r(J)$.

(ii) If $J^{\prime}(\neq J)$ is a critical sub-piece of $J$ and is also a pullback of $J$, then for $k$ the number of red spots on the half open diagonal $\left[J^{\prime} /^{J}[\right.$, we have

$$
\begin{gathered}
r(J) \leq\left.\right|_{J^{\prime}} /^{J} \mid \leq k \cdot r\left(J^{\prime}\right) . \\
\left.\begin{array}{c}
R(J)< \\
2 \cdot r(J) \leq
\end{array}\right\}||_{\Gamma(J)} /^{J} \mid \leq r(\Gamma(J)), \text { in particular } r(\Gamma(J)) \geq 2 r(J) .
\end{gathered}
$$

(iv) For any integer $\tau \geq 1$, let $\Gamma^{\tau}(J)$ be the $\tau$-th generation of last successors,

$$
\begin{gathered}
\left.2^{\tau-1}\right|_{\Gamma(J)} /^{J} \mid \leq r\left(\Gamma^{\tau}(J)\right) ; \\
\forall \text { critical piece } L \supset J, \quad 2^{\tau} r(L) \leq 2^{\tau} r(J) \leq r\left(\Gamma^{\tau}(J)\right) ; \\
\left(2^{\tau+1}-2\right) r(J) \leq\left.\right|_{\Gamma^{\tau}(J)} /^{J} \mid \leq 2 r\left(\Gamma^{\tau}(J)\right) .
\end{gathered}
$$

Proof. (i) Obvious, by Rule 1 .

(ii) Denote by $T$ the upper triangle with vertices $J^{\prime}, J, J$. Then $\left|J^{\prime} /^{J}\right|$ is also the length of the top edge of $T$. Consequently $\left|J^{\prime} /{ }^{J}\right| \geq r(J)$.

Let $c^{\prime} \in \operatorname{Crit}(f)$ and $E, F$ be two consecutive red spots on $\operatorname{row}\left(J^{\prime}\right)$ of $\mathcal{T}\left(c^{\prime}\right)$ with $F$ on the right of $E$. By Rule 2 the triangle $T$ appears from $\operatorname{column}(E)$ with $E$ as the lower vertex.

Assume at first $k=1$, i.e. $]_{J^{\prime}} /^{J[}$ contains no red spots. Applying Rule 1 one sees that the length of the horizontal segment $E-F$ must be at least $\left|J_{J^{\prime}} /^{J}\right|$. Therefore $\left|J_{J^{\prime}} /^{J}\right| \leq r\left(J^{\prime}\right)$.

In the general case, let $\left(J^{\prime}=\right) J^{k}, J^{k-1}, \cdots, J^{1}, J^{0}(=J)$ be the consecutive red spots on the closed diagonal $\left[J^{\prime} /^{J}\right.$. Applying the above argument on each $\left[J^{l+1} / J^{l}[\right.$ we get

$$
\left|J^{\prime} /^{J}\right| \leq r\left(J^{k}\right)+r\left(J^{k-1}\right)+\cdots+r\left(J^{1}\right) \stackrel{(\mathrm{i})}{\leq} k \cdot r\left(J^{k}\right)=k \cdot r\left(J^{\prime}\right) .
$$

(iii) Fix any $c^{\prime} \in C r i t(f)$ (with $c^{\prime}$ may or may not be $\mathbf{c}$ ). 
In $\mathcal{T}\left(c^{\prime}\right)$, let $J-J$ be a horizontal segment bounded by two consecutive red spots on row $(J)$. Form an upper triangle $T$ with this segment as the upper edge. Denote its lower vertex by $W$, and its length by $l$.

If $W$ is critical, it has to be red since $W$ is below $J$. Now use Rule 2 to compare $T$ with the upper triangle in $\mathcal{T}(\mathbf{c})$ with left edge ${ }_{\mathcal{L}_{\mathbf{c}}(J)}^{J}$ and (automatically) right vertex $J$, one concludes that $W=\mathcal{L}_{\mathbf{c}}(J)$ and $W$ is the first successor of $J$. But there are at least two successors, so $l<\left|{ }_{\Gamma(J)} /^{J}\right|$.

If $W$ is not critical, follow its left-down diagonal in $\mathcal{T}\left(c^{\prime}\right)$ until the first critical vertex $W^{\prime}$ (such $W^{\prime}$ exists since the 0 -th column vertex on that diagonal is critical).

Now by the hypothesis of recurrence, $W^{\prime}$ appears on $\operatorname{row}\left(W^{\prime}\right)$ of $\mathcal{T}(\mathbf{c})$. Therefore $\widehat{\mathcal{L}}_{\mathbf{c}}\left(W^{\prime}\right)$ exists and is a successor of $J$. It follows that $l<\left.\right|_{\widehat{\mathcal{L}}_{\mathbf{c}}\left(W^{\prime}\right)} /{ }^{J}|\leq|_{\Gamma(J)} /{ }^{J} \mid$.

This proves $R(J)<\left.\right|_{\Gamma(J)} /^{J} \mid$.

By the definition of a successor, the half open diagonal $\left[\Gamma(J) /{ }^{J}[\right.$ contains only one red spot: $\Gamma(J)$. So $\left.\right|_{\Gamma(J)} /^{J} \mid \leq r(\Gamma(J))$ by (ii). Denote by $T_{\Gamma}$ the upper triangle with vertices $\Gamma(J), J, J$. As $J$ has at least two successors (Lemma 4.2) and $\Gamma(J)$ is the last successor, the top edge of $T_{\Gamma}$ contains at least three red spots (counting the ends). Therefore $2 \cdot r(J) \leq\left|T_{\Gamma}\right|=\left|{ }_{\Gamma(J)} /^{J}\right|$.

(iv) We claim at first

$$
2^{j} r(J) \leq\left|\Gamma^{j}(J) /^{\Gamma^{j-1}(J)}\right| \leq \frac{1}{2^{\tau-j}} r\left(\Gamma^{\tau}(J)\right) \text { for } j=1, \cdots, \tau
$$

this follows from applying recursively (iii):

$$
\left|\Gamma^{j}(J) /^{\Gamma^{j-1}(J)}\right| \geq 2 r\left(\Gamma^{j-1}(J)\right) \geq 2^{2} r\left(\Gamma^{j-2}(J)\right) \geq \cdots \geq 2^{j} r(J),
$$

and

$$
\left|\Gamma^{j}(J) /^{\Gamma^{j-1}(J)}\right| \leq r\left(\Gamma^{j}(J)\right) \leq \frac{1}{2} r\left(\Gamma^{j+1}(J)\right) \leq \cdots \leq \frac{1}{2^{\tau-j}} r\left(\Gamma^{\tau}(J)\right) .
$$

Now the case $j=1$ of $(* * *)$ gives $\left.2^{\tau-1}\right|_{\Gamma(J)} /^{J} \mid \leq r\left(\Gamma^{\tau}(J)\right)$, i.e. (7). Combining this with (i) and (iii) we get (지 $)$. To get (9), we just need to sum up (***) for $j=1, \cdots, \tau:\left(2^{\tau+1}-2\right) r(J) \leq\left|\Gamma_{\Gamma^{\tau}(J)} /^{J}\right|=\left.\sum_{j=1}^{\tau}\right|_{\Gamma^{j}(J)} /^{\Gamma^{j-1}(J)} \mid \leq 2 r\left(\Gamma^{\tau}(J)\right)$.

\subsection{The operators $\mathcal{A}$ and $\mathcal{B}$.}

Lemma 4.4. Fix any $\mathbf{c} \in$ Crit $(f)$, say colored red. Let I be any puzzle piece containing $\mathbf{c}$. Then row $(I)$ of $\mathcal{T}(\mathbf{c})$ admits two consecutive $I$ vertices, the first one being on the $t$-th column for some $t>0$ (and minimal possible), leading diagonally to two critical pieces $\mathcal{B}(I), \mathcal{A}(I)$ on the 0 -th column, such that $\underset{\mathcal{A}(I)}{\mathcal{B}(I)}$ is vacuous; and

$$
\left\{\begin{array} { l } 
{ \operatorname { d e g } ( \mathcal { B } ( I ) / ^ { I } ) \leq \delta ^ { b ^ { 2 } } = : C _ { \mathcal { B } } ; } \\
{ 1 \leq \# ( \{ \text { red spots } \} \cap [ \mathcal { B } ( I ) / ^ { I } [ ) \leq b ; }
\end{array} \left\{\begin{array}{l}
\operatorname{deg}\left(\mathcal{A}_{(I)} /{ }^{I}\right) \leq \delta^{b^{2}+b}=: C_{\mathcal{A}} \\
1 \leq \#\left(\{ \text { red spots } \} \cap \left[\mathcal{A}(I) /^{I}[) \leq b+1\right.\right.
\end{array}\right.\right.
$$


The proof will be postponed to Appendix C A simpler proof in the case $b=1$ can be found in Appendix D. The following consequence of this lemma will be frequently used in the sequel:

$$
\begin{gathered}
C_{\mathcal{B}}<C_{\mathcal{A}}, \quad r(I) \leq\left.\right|_{\mathcal{A}(I)} ^{\mathcal{B}(I)} \mid \leq R(I) ; \\
r(I) \leq||_{\mathcal{B}(I)} /^{I} \mid \leq b \cdot r(\mathcal{B}(I)) \quad \text { and } \quad 2 r(I) \leq\left.\right|_{\mathcal{A}(I)} /^{I} \mid \leq(b+1) \cdot r(\mathcal{A}(I)) . \\
\hline
\end{gathered}
$$

Proof. The length $|\underset{\mathcal{A}(I)}{\mathcal{B}(I)}|$ is equal to the length of a horizontal segment on $\operatorname{row}(J)$ of $\mathcal{T}(\mathbf{c})$ linking two consecutive $I$ vertices. It is therefore bounded between $r(I)$ and $R(I)$. Now ${ }_{[\mathcal{B}(I)} /^{I]}$ (respectively ${ }_{[\mathcal{A}(I)} /^{I]}$ ) is the edge of an upper triangle whose upper edge contains at least two (respectively three) $I$ vertices, its length is therefore at least $r(I)$ (respectively $2 r(I)$ ). The rest follows from Corollary 4.3(ii) (for $k=b, b+1$, respectively).

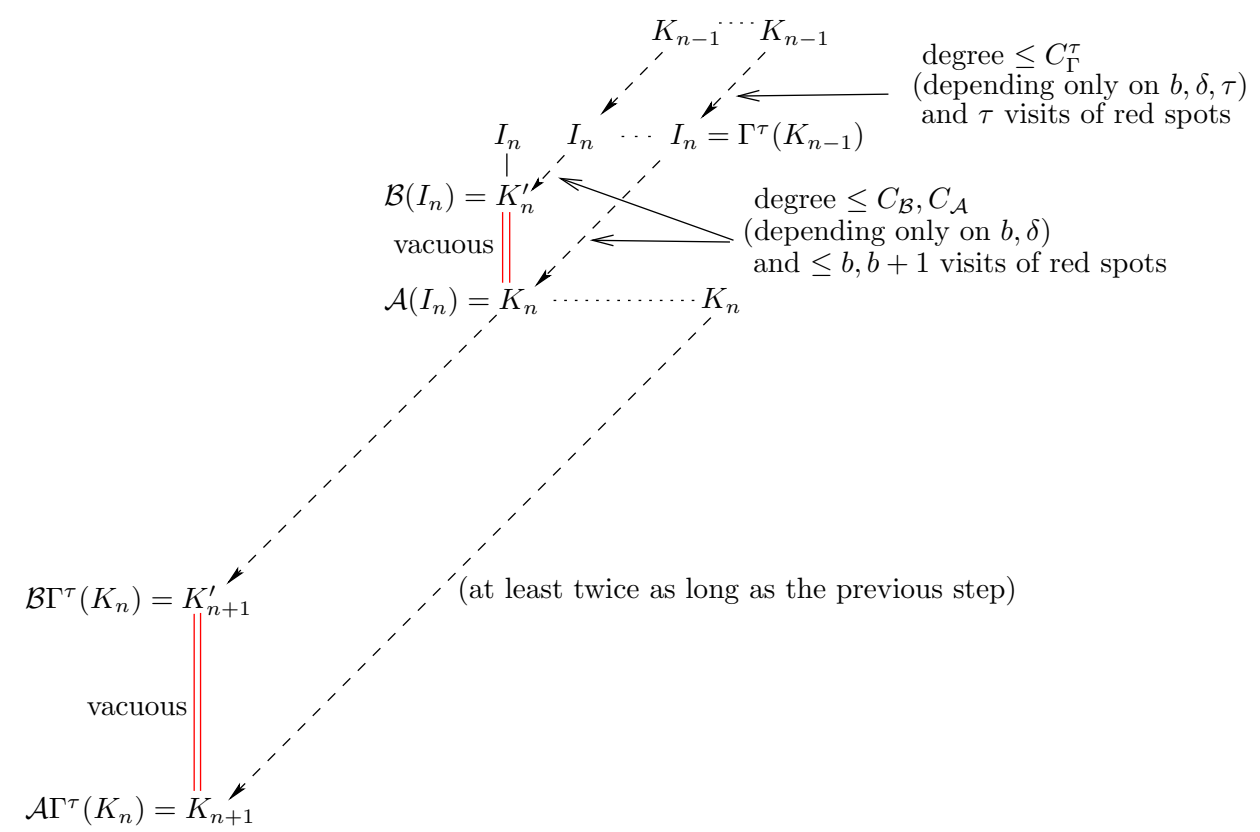

Figure 5. The operators $\mathcal{A}, \mathcal{B}, \Gamma^{\tau}$ and the short cutted KSS nest.

4.5. Proposition 3.2 with the short cutted KSS nest. Fix $\tau \geq 1$ an integer. Recall that $\Gamma^{\tau}$ denotes the $\tau$-th generation of last successors. Applying repeatedly Lemma 4.2(b), we get, for any critical piece $J, \operatorname{deg}\left(\Gamma^{\tau}(J) /^{J}\right) \leq C_{\Gamma}^{\tau}$.

Fix $\mathbf{c} \in C r i t(f)$ and fix $K_{0}$ a critical puzzle piece of $\mathbf{c}$. For $n \geq 1$, we define inductively $K_{n}^{\prime}:=\mathcal{B} \Gamma^{\tau}\left(K_{n-1}\right)$ and $K_{n}:=\mathcal{A} \Gamma^{\tau}\left(K_{n-1}\right)$; see Figure 5 , 
Set $C_{K}=C_{\mathcal{A}} \cdot C_{\Gamma}^{\tau}$ and $\beta=C_{\mathcal{B}} \cdot C_{\Gamma}^{\tau} \cdot C_{K}$. They depend only on $b, \delta$. It follows directly from Lemmas 4.2 and 4.4 that, $\forall n$,

$$
\operatorname{deg}\left(K_{n+1}^{\prime} /^{K_{n-1}}\right)=\operatorname{deg}\left(K_{n+1}^{\prime} /^{K_{n}}\right) \cdot \operatorname{deg}\left(K_{n} /^{K_{n-1}}\right) \leq C_{\mathcal{B}} \cdot C_{\Gamma}^{\tau} \cdot C_{K}=\beta .
$$

Set $I_{n}=\Gamma^{\tau}\left(K_{n-1}\right)$. Then $K_{n}^{\prime}=\mathcal{B}\left(I_{n}\right)$ and $K_{n}=\mathcal{A}\left(I_{n}\right)$; see Figure 5 ,

Lemma 4.5. We have, $\forall n$,

$$
\begin{gathered}
2^{\tau} r\left(I_{n}\right) \leq r\left(I_{n+1}\right) ; \\
\left(2^{\tau+2}-2\right) r\left(K_{n}\right) \leq\left.\right|_{K_{n+1}} /^{K_{n}}|\leq 2|_{K_{n+1}} /^{I_{n+1}} \mid ; \\
\mid K_{n} /^{K_{n-1} \mid} \leq(b+3) r\left(K_{n}\right) .
\end{gathered}
$$

Proof. Formula (14) follows from (8) applied to the triple $I_{n}, K_{n}, \Gamma^{\tau}\left(K_{n}\right)$.

Set $u_{n}=\left.\right|_{K_{n}=\mathcal{A}\left(I_{n}\right)} /^{I_{n}}\left|, \quad p_{n}=\right|_{K_{n}} /^{K_{n-1}}\left|, \quad q_{n}=\right|_{I_{n+1}=\Gamma^{\tau}\left(K_{n}\right)} /^{K_{n}} \mid$.

Applying (91), (8) and (10) we get the following bounds for $q_{n}$ and $u_{n}$ for all $n$ :

$$
\begin{gathered}
\left(2^{\tau+1}-2\right) r\left(K_{n}\right) \leq q_{n} \leq 2 r\left(I_{n+1}\right) \leq 2 r\left(K_{n+1}\right) ; \\
2^{\tau+1} r\left(K_{n-1}\right) \leq 2 r\left(I_{n}\right) \leq u_{n} \leq(b+1) r\left(K_{n}\right) .
\end{gathered}
$$

Combining these we get

$$
\begin{gathered}
p_{n+1}=q_{n}+u_{n+1} \geq\left(2^{\tau+1}-2\right) r\left(K_{n}\right)+2^{\tau+1} r\left(K_{n}\right)=\left(2^{\tau+2}-2\right) r\left(K_{n}\right): \\
p_{n}=q_{n-1}+u_{n} \leq 2 r\left(K_{n}\right)+(b+1) r\left(K_{n}\right)=(b+3) r\left(K_{n}\right) ; \\
p_{n+1}=q_{n}+u_{n+1} \leq 2 r\left(I_{n+1}\right)+u_{n+1} \leq 2 u_{n+1} .
\end{gathered}
$$

Proof of Proposition 3.2. Fix $\tau$ to be any integer no less than $b+1$ (actually $\tau=b$ will be also suitable, see the remark below). We are going to prove Proposition 3.2 for our double sequence $\left(K_{n}^{\prime}, K_{n}\right)_{n}$. At first, due to $\left.\right|_{K_{n}} /^{K_{0}} \mid=p_{n}+p_{n-1}+\cdots+p_{1}$ and

$$
2 p_{n} \stackrel{16}{\leq} 2(b+3) r\left(K_{n}\right) \stackrel{\tau \geq b+1}{\leq}\left(2^{\tau+2}-2\right) r\left(K_{n}\right) \stackrel{15}{\leq} p_{n+1}
$$

we have

$$
\forall n, \quad 2 p_{n} \leq p_{n+1} \text { and }\left|K_{n} / K^{K_{0}}\right|<2 p_{n} .
$$

The fact that $\left(K_{n}^{\prime}, K_{n}\right)_{n}$ satisfies (2) is due to the construction and (12).

Point (a) is simply (11).

Point (b), i.e. $\operatorname{deg}\left(B^{\prime} /^{K_{m}^{\prime}}\right) \leq \alpha$. By the definition of $B$ we have $B=\widehat{\mathcal{L}}_{\hat{y}}\left(K_{m}\right)$. Now formula (5) applied to $B$ together with the fact that $\left(K_{m}^{\prime} \backslash \bar{K}_{m}\right) \cap \widehat{\mathcal{P}}_{f}=\emptyset$ imply that $\operatorname{deg}\left(B^{\prime} /^{K_{m}^{\prime}}\right)=\operatorname{deg}\left(B /{ }^{K_{m}}\right) \leq \alpha$.

Point (d), i.e $\operatorname{deg}\left(K_{m+2}^{\prime} / K_{m}\right) \leq \beta$ is due to (13).

The proof of Points (c): $\operatorname{deg}\left(A^{\prime} / B^{\prime}\right) \leq d$ and (e): $f^{\xi}\left(K_{m+2}\right) \subset A$, will depend on the value of $\tau$, the formulae (15), (16), $u_{m}=\left.\right|_{K_{m}=\mathcal{A}\left(I_{m}\right)} /^{I_{m}} \mid$ and Lemma 4.4

See Figure 6 for the following construction. Starting from $\mathcal{T}(\hat{x})$, we define inductively the sequence of column numbers $\kappa_{j}$ as follows: set $\kappa_{0}=0$. From column 


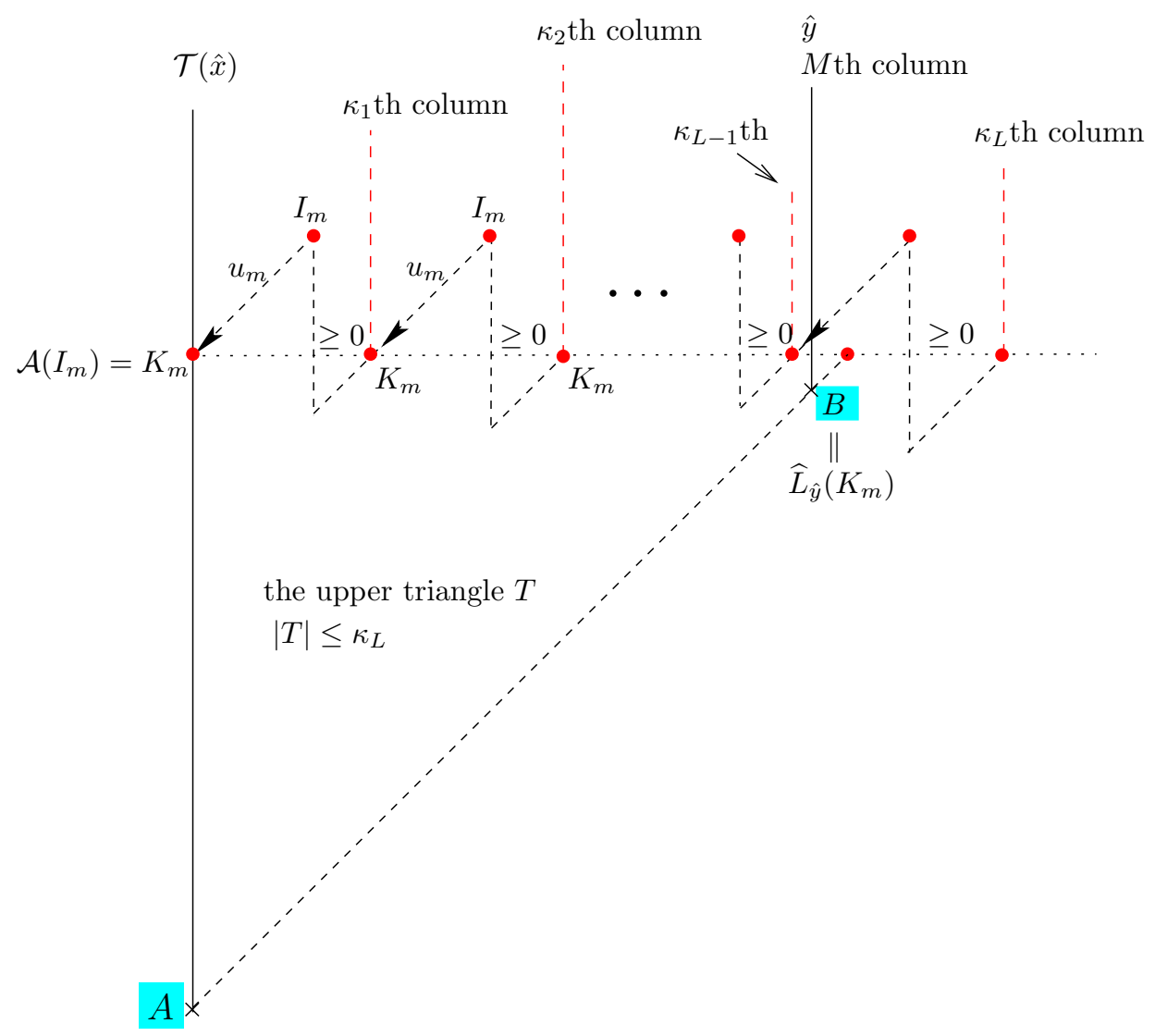

Figure 6. Control of $\operatorname{deg}\left({ }_{A} /^{B}\right)$ and \# $\#$ red spots $\}$ on the top of $T$

$\kappa_{j}$, row $\left(K_{m}\right)$, march at first $u_{m}$ steps to the right, then march to the right until the first $\geq 0$ hit of a red spot, and set $\kappa_{j+1}$ to be the corresponding column number. Let now $L$ be the minimal integer so that $\kappa_{L}>M:=\left|K_{m} /{ }^{K_{0}}\right|$.

Therefore $\kappa_{L-1} \leq M$. Then $(L-1) \cdot u_{m} \leq \kappa_{L-1} \leq M \stackrel{18}{<} 2 p_{m} \stackrel{15}{\leq} 4 u_{m}$. Consequently

$$
L \leq 4
$$

Now $\operatorname{deg}\left({ }_{A} /^{B}\right) \leq\left(\operatorname{deg}\left(K_{m} /{ }^{I_{m}}\right) \cdot \alpha\right)^{L}=\left(\operatorname{deg}\left({\mathcal{A}\left(I_{m}\right)}^{I_{m}}\right) \cdot \alpha\right)^{L} \leq\left(C_{\mathcal{A}} \cdot \alpha\right)^{4}=: d$. But $\operatorname{deg}\left(A /^{B}\right)=\operatorname{deg}\left(A^{\prime} /^{B^{\prime}}\right)$ (as $K_{m}^{\prime} \backslash \bar{K}_{m}$ contains no postcritical points), this gives Point (c).

Point (e). For this we will estimate the number of red spots on the top edge of the upper triangle $T^{\prime}$ (respectively $T$ ), defined so to have a left edge $\begin{gathered}K_{m} \\ \mid \\ f^{\xi}\left(K_{m+2}\right)\end{gathered}$ (respectively $\left.\begin{array}{c}K_{m} \\ \mid \\ A\end{array}\right)$ on $\operatorname{column}(\hat{x})$. 
At first, recall that $B=\widehat{\mathcal{L}}_{\hat{y}}\left(K_{m}\right)$, so $\kappa_{L}$ is actually greater than or equal to the column number (in $\mathcal{T}(\hat{x})$ ) of the right vertex of $T$. Also, by Rule 1 , the number of red spots on row $\left(K_{m}\right)$ from the $\kappa_{j}$-th column (excluded) to the $\kappa_{j+1}$-th column (included) is at most equal to the number of red spots on $] K_{m}=\mathcal{A}\left(I_{m}\right) /{ }^{\left.I_{m}\right]}$, which is, by Lemma 4.4, at most $b+1$. Therefore, denoting by $\operatorname{top}(T)$ the top edge of $T$ including the end vertices,

$$
\#\{\text { red-spots } \in \operatorname{top}(T)\}-1 \stackrel{\text { def. of } L}{\leq} L \cdot(b+1) \stackrel{19}{\leq} 4(b+1) .
$$

On the other hand,

$$
\begin{aligned}
& \left|\Gamma_{\left(K_{m}\right)} / K_{m}\right| \cdot\left(\#\left\{\text { red-spots } \in \operatorname{top}\left(T^{\prime}\right)\right\}-1\right) \\
> & R\left(K_{m}\right) \cdot\left(\#\left\{\text { red-spots } \in \operatorname{top}\left(T^{\prime}\right)\right\}-1\right) \\
= & R\left(K_{m}\right) \cdot\left(\#\left\{\text { segments on } \operatorname{top}\left(T^{\prime}\right) \text { linking two consecutive red-spots }\right\}\right) \\
(21) & \stackrel{*}{\geq} \text { sum of the lengths of these segments }=\left|T^{\prime}\right| \\
& \stackrel{* *}{\geq} r\left(I_{m+2}\right)=\frac{r\left(I_{m+2}\right)}{r\left(I_{m+1}\right)} r\left(I_{m+1}\right) \stackrel{14}{\geq} 2^{\tau} r\left(I_{m+1}\right)=2^{\tau} r\left(\Gamma^{\tau}\left(K_{m}\right)\right) \\
& \quad 7 \\
& \geq\left. 2^{\tau} \cdot 2^{\tau-1}\right|_{\Gamma\left(K_{m}\right)} / K_{m}\left|=2^{2 \tau-1} \cdot\right| \Gamma_{\left(K_{m}\right)} / K_{m} \mid,
\end{aligned}
$$

where the first inequality is due to Corollary 4.3(iii); the one marked by $*$ is due to the definition of $R\left(K_{m}\right)$ as the maximal possible length between two consecutive red spots on row $\left(K_{m}\right)$; the one marked by $* *$ is due to the fact that the left edge of $T^{\prime}$ forms a parallelogram with $\underset{K_{m+2}}{K_{m+2}^{\prime}}$ (see Figure 3]), therefore $\left|T^{\prime}\right|=\left|\underset{K_{m+2}}{\mid K_{m+2}^{\prime}}\right|=$ $\left|\begin{array}{c}\mathcal{B}\left(I_{m+2}\right) \\ \mid \\ \mathcal{A}\left(I_{m+2}\right)\end{array}\right|$, which in turn is greater than or equal to $r\left(I_{m+2}\right)$ by (10). Therefore

$$
\text { \# }\left\{\text { red-spots } \in \operatorname{top}\left(T^{\prime}\right)\right\}-1 \geq 2^{2 \tau-1} .
$$

Notice that for $b \geq 1$, we have $2^{2 \tau-1} \geq 4(b+1)$ (recall that $\left.\tau=b+1\right)$. Therefore (22) and (20) together imply that $f^{\xi}\left(K_{m+2}\right) \subset A$. This is Point (e).

Finally $\operatorname{depth}\left(K_{n}\right) \geq \operatorname{depth}\left(K_{n}\right)-\operatorname{depth}\left(K_{n-1}\right)=p_{n} \stackrel{18}{\geq} 2^{n-1} p_{1}$, and

$$
\operatorname{depth}\left(K_{n}\right)-\operatorname{depth}\left(K_{n}^{\prime}\right)=\left|\underset{K_{n}}{K_{n}^{\prime}}\right|=\left|\underset{\mathcal{A}\left(I_{n}\right)}{\mathcal{B}\left(I_{n}\right)}\right| \stackrel{10}{\geq} r\left(I_{n}\right) \stackrel{14}{\geq} 2^{\tau(n-1)} r\left(I_{1}\right) .
$$

Therefore all these depth sequences grow exponentially fast.

Remark 1. The number $\tau=b+1$ is chosen to be the least integer to satisfy both $2^{\tau+2}-2 \geq 2(b+3)$ (see (17) $)$ and $2^{2 \tau-1} \geq 4(b+1)$. Actually one can also take $\tau=b$, and use the above proof in case $b \geq 2$ and another set of estimates in case $b=1$ (see Appendix D).

4.6. Proposition 3.2 for the original KSS nest. Fix an integer $\tau \geq 1$ (which will be $\geq b+2)$.

Fix $\mathbf{c} \in \operatorname{Crit}(f)$ and fix $K_{0}$ a critical puzzle piece of c. For $n \geq 1$, we define inductively $K_{n}=\mathcal{B} \mathcal{A} \Gamma^{\tau}\left(K_{n-1}\right)$ and $K_{n}^{\prime}$ to be the puzzle piece so that $\begin{gathered}K_{n}^{\prime} \\ K_{n}\end{gathered}$ forms a parallelogram with $\begin{gathered}\mathcal{B} \Gamma^{\tau}\left(K_{n-1}\right) \\ \underset{\mathcal{A} \Gamma^{\tau}\left(K_{n-1}\right)}{\mid}\end{gathered}$. This time, set $C_{K}=C_{\mathcal{B}} \cdot C_{\mathcal{A}} \cdot C_{\Gamma}^{\tau}$ and $\beta=$ 
$C_{\mathcal{B}}^{2} \cdot C_{\Gamma}^{\tau} \cdot C_{K}$. They depend only on $b, \delta$. It follows from Lemmas 4.2 and 4.4 that:

$$
\forall n, \quad\left(K_{n}^{\prime} \backslash K_{n}\right) \cap \widehat{\mathcal{P}}_{f}=\emptyset, \quad \operatorname{deg}\left(K_{n} / K^{K_{n-1}}\right) \leq C_{\mathcal{B}} \cdot C_{\mathcal{A}} \cdot C_{\Gamma}^{\tau}=C_{K} .
$$

Notice that $\operatorname{deg}\left(K_{n}^{\prime} / \mathcal{B}\left(I_{n}\right)\right)=\operatorname{deg}\left(K_{n} /{ }^{L_{n}}\right)$, due to the vacuousness of $\begin{gathered}\mathcal{B}\left(I_{n}\right) \\ I_{n} \\ L_{n}\end{gathered}$. Therefore $\operatorname{deg}\left(K_{n}^{\prime} /{ }^{K_{n-1}}\right) \leq C_{\mathcal{B}} C_{\mathcal{B}} C_{\Gamma}^{\tau}<C_{\mathcal{B}} C_{\mathcal{A}} C_{\Gamma}^{\tau}=C_{K}$, and

$$
\operatorname{deg}\left(K_{n+1}^{\prime} /^{K_{n-1}}\right)=\operatorname{deg}\left(K_{n+1}^{\prime} /^{K_{n}}\right) \cdot \operatorname{deg}\left(K_{n} /{ }^{K_{n-1}}\right) \leq C_{\mathcal{B}}^{2} \cdot C_{\Gamma}^{\tau} \cdot C_{K}=\beta .
$$

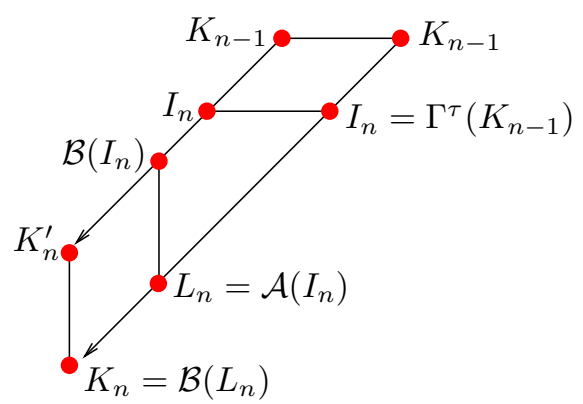

We are going to prove Proposition 3.2 for this new sequence $\left(K_{n}^{\prime}, K_{n}\right)_{n}$.

Definition. Set $\forall n \geq 1, I_{n}=\Gamma^{\tau}\left(K_{n-1}\right)$ and $L_{n}=\mathcal{A}\left(I_{n}\right)$. Then $K_{n}=\mathcal{B}\left(L_{n}\right)$.

Let now

$q_{n}=\left.\right|_{I_{n+1}=\Gamma^{\tau}\left(K_{n}\right)} /^{K_{n}}\left|, s_{n}=\right|_{L_{n}=\mathcal{A}\left(I_{n}\right)} /^{I_{n}}\left|, t_{n}=\right|_{K_{n}=\mathcal{B}\left(L_{n}\right)} /^{L_{n}}\left|, p_{n}=\right|_{K_{n}} /^{K_{n-1}} \mid$.

Here we still have (14), i.e. $2^{\tau} r\left(I_{n}\right) \leq r\left(I_{n+1}\right)\left(\right.$ as $2^{\tau} r\left(K_{n}\right) \leq r\left(\Gamma^{\tau}\left(K_{n}\right)\right)=$ $r\left(I_{n+1}\right)$ by (8) and $\left.r\left(I_{n}\right) \leq r\left(K_{n}\right)\right)$. Furthermore, we have

$$
\begin{gathered}
p_{n} \leq(b+4) t_{n} ; \\
\tau \geq b+2 \Longrightarrow \quad p_{n} \geq 2 p_{n-1} \text { and } p_{n}+p_{n-1}+\cdots+p_{1}<2 p_{n} .
\end{gathered}
$$

Proof. Corollary 4.3 together with (10) give easily the bounds for $q_{n}, s_{n}$ and $t_{n}$ :

$$
\begin{gathered}
\left(2^{\tau+1}-2\right) r\left(K_{n}\right) \leq q_{n} \leq 2 \cdot r\left(I_{n+1}\right) \leq 2 \cdot r\left(L_{n+1}\right) \leq 2 \cdot r\left(K_{n+1}\right) ; \\
2^{\tau+1} r\left(K_{n-1}\right) \leq 2 r\left(I_{n}\right) \leq s_{n} \leq(b+1) \cdot r\left(L_{n}\right) \leq(b+1) \cdot r\left(K_{n}\right) ; \\
2^{\tau} r\left(K_{n-1}\right) \leq r\left(I_{n}\right) \leq r\left(L_{n}\right) \leq t_{n} \leq b \cdot r\left(K_{n}\right) .
\end{gathered}
$$

Combining the three we get

$$
\begin{gathered}
p_{n+1}=q_{n}+t_{n+1}+s_{n+1} \geq\left(2^{\tau+2}+2^{\tau}-2\right) r\left(K_{n}\right) ; \\
p_{n}=q_{n-1}+s_{n}+t_{n} \leq(2+(b+1)+b) r\left(K_{n}\right)=(2 b+3) r\left(K_{n}\right) ; \\
p_{n} \leq 2 \cdot r\left(L_{n}\right)+(b+1) r\left(L_{n}\right)+t_{n}=(b+3) r\left(L_{n}\right)+t_{n} \leq(b+4) t_{n} .
\end{gathered}
$$

Therefore $2 p_{n} \leq 2(2 b+3) r\left(K_{n}\right) \stackrel{\tau \geq b+2}{\leq}\left(2^{\tau+2}+2^{\tau}-2\right) r\left(K_{n}\right) \leq p_{n+1}$.

New proof of Proposition 3.2. We take $\tau \geq b+2$ and construct the KSS nest as above. 
We follow the same line as in $\$ 4.5$. Points (a),(b) and (d) are proved as before. Use $\left(t_{m}, \mathcal{B}, L_{m}\right)$ instead of $\left(u_{m}, \mathcal{A}, I_{m}\right)$ to define the sequence $\kappa_{j}$. Define $L$ as before so that $\kappa_{L}>M:=\left|K_{m} / K_{0}\right|=p_{m}+p_{m-1}+\cdots+p_{1}$. Then

$$
(L-1) \cdot t_{m} \leq \kappa_{L-1} \leq M \stackrel{25}{\stackrel{25}{<}} 2 p_{m} \stackrel{24}{\leq} 2(b+4) \cdot t_{m} .
$$

Consequently $L \leq 2(b+4)$. Point (c) follows, for $d=\left(C_{\mathcal{B}} \cdot \alpha\right)^{2(b+4)}$. Now

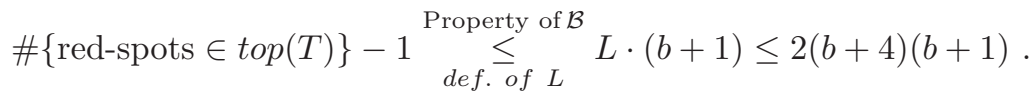

The other part \#\{red-spots $\left.\in \operatorname{top}\left(T^{\prime}\right)\right\}-1 \geq 2^{2 \tau-1}$ is proved in the same way as in 4.5 . Now Point (e) follows from $2^{2 \tau-1} \geq 2(b+4)(b+1)($ as $\tau \geq b+2)$.

For any $n \geq 2$, set $K_{n}^{-}=\mathcal{A}\left(L_{n}\right)$ and denote by $Q_{n+1}$ to be the puzzle piece containing $\mathbf{c}$ so that $\underset{Q_{n+1}}{K_{n+1}}$ and $\underset{K_{n}^{-}}{K_{n}}$ form a parallelogram 2 (See Figure 7 )

Corollary 4.7. For every $n \geq 2$, both $\underset{K_{n}^{-}}{K_{n}}$ and $\underset{Q_{n+1}}{K_{n+1}}$ are vacuous, $\left(K_{n+1}^{-} \cup\right.$ $\left.\bigcup_{x \in \widehat{\mathcal{P}}_{f} \cap K_{n+1}} \mathcal{L}_{x}\left(K_{n+1}\right)\right) \subset Q_{n+1}$, and

$$
\inf _{x \in \widehat{\mathcal{P}}_{f} \cap K_{n+1}} \bmod \left(K_{n+1} \backslash \overline{\mathcal{L}_{x}\left(K_{n+1}\right)}\right) \geq \frac{\bmod \left(K_{n} \backslash \overline{K_{n}^{-}}\right)}{C_{K}} \geq \frac{\bmod \left(K_{n-1}^{\prime} \backslash \bar{K}_{n-1}\right)}{C_{K} C_{\mathcal{A}}^{2} C_{\Gamma}^{\tau}} .
$$

Furthermore,

$$
\left|\underset{K_{n+1}^{-}}{K_{n+1}}\right|>2^{\tau-1}\left|\underset{K_{n}^{-}}{K_{n}}\right| ;\left|\underset{K_{n+1}}{\mid K_{n+1}^{\prime}}\right|>2^{\tau-1}\left|\underset{K_{n}}{\mid K_{n}^{\prime}}\right|
$$

Proof. The vacuousness follows from the property of $\mathcal{A}$ and $\mathcal{B}$ (Lemma 4.4). Now

$$
\begin{aligned}
& r\left(I_{n+1}\right)=\left.r\left(\Gamma^{\tau}\left(K_{n}\right)\right) \stackrel{17}{\geq} 2^{\tau-1}\right|_{\Gamma\left(K_{n}\right)} /\left.K_{n}\right|_{\text {Corollary 4.3 }} ^{>} 2_{\text {iii })}^{\tau-1} R\left(K_{n}\right) \\
& \geq\left\{\begin{array}{l}
2^{\tau-1} R\left(L_{n}\right) \stackrel{10}{\geq} 2^{\tau-1}\left|\underset{\mathcal{A}\left(L_{n}\right)}{\mathcal{B}\left(L_{n}\right)}\right|=2^{\tau-1}\left|\underset{K_{n}^{-}}{\mid K_{n}}\right| \geq\left|\underset{K_{n}^{-}}{\mid}\right|=\left|\underset{Q_{n+1}}{K_{n}}\right|, \\
2^{\tau-1} R\left(I_{n}\right) \geq 2^{\tau-1}\left|\begin{array}{c}
\mathcal{B}\left(I_{n}\right) \\
\mid \\
\mathcal{A}\left(I_{n}\right)
\end{array}\right|=2^{\tau-1}\left|\underset{K_{n}}{\mid K_{n}^{\prime}}\right| \geq\left|\underset{K_{n}}{\mid K_{n}}\right| .
\end{array}\right.
\end{aligned}
$$

Hence $\left|\underset{K_{n+1}^{-}}{K_{n+1}}\right| \geq r\left(L_{n+1}\right) \geq r\left(I_{n+1}\right)>2^{\tau-1}\left|\underset{K_{n}^{-}}{\mid K_{n}}\right| ;\left|\underset{K_{n+1}}{\mid K_{n+1}^{\prime}}\right| \geq r\left(I_{n+1}\right)>2^{\tau-1}\left|\underset{K_{n}}{I_{n}^{\prime}}\right|$. And, for any $x \in \widehat{\mathcal{P}}_{f} \cap K_{n+1},\left|\underset{\mathcal{L}_{x}\left(K_{n+1}\right)}{K_{n+1}}\right| \geq r\left(K_{n+1}\right) \geq r\left(I_{n+1}\right) \geq\left|\underset{Q_{n+1}}{K_{n+1}}\right|$. Similarly

$$
\begin{aligned}
& \left|\underset{K_{n}^{-}}{K_{n}}\right| \geq r\left(L_{n}\right) \geq r\left(I_{n}\right)=r\left(\Gamma^{\tau}\left(K_{n-1}\right)\right) \stackrel{8}{\geq} 2^{\tau} r\left(K_{n-1}\right) \\
& \stackrel{10}{\geq} 2^{\tau} \frac{\mid K_{n-1}=\mathcal{B}\left(L_{n-1}\right)}{b^{L_{n-1}} \mid}>\left|K_{n-1} /^{L_{n-1}}\right|>\left|\underset{K_{n-1}}{K_{n-1}^{\prime}}\right| .
\end{aligned}
$$

\footnotetext{
${ }^{2}$ The definition of $K_{n}^{-}$and the following estimate about $\bmod \left(K_{n} \backslash \overline{K_{n}^{-}}\right)$appeared in $\mathrm{YZ}$ for the first time.
} 
Let $J$ be the puzzle piece so that $\underset{K_{n}^{-}}{J}$ and $\begin{gathered}K_{n-1}^{\prime} \\ K_{n-1}\end{gathered}$ form a parallelogram. The above length calculation shows that $J \subset K_{n}$. Fix any $x \in \widehat{\mathcal{P}}_{f} \cap K_{n+1}$. We know that $Q_{n+1}$ appear on the 0 -th column of $\mathcal{T}(x)$, due to the vacuousness of $\begin{gathered}K_{n+1} \\ \mid \\ Q_{n+1}\end{gathered}$. Then by the above calculation we have $\mathcal{L}_{x}\left(K_{n+1}\right) \subset Q_{n+1}$. Similarly $K_{n+1}^{-} \subset Q_{n+1}$. Finally

$$
\begin{aligned}
\bmod \left(K_{n} \backslash \overline{K_{n}^{-}}\right) \geq \bmod \left(J \backslash \overline{K_{n}^{-}}\right) & \stackrel{\text { vacuous }}{=} \frac{\bmod \left(K_{n-1}^{\prime} \backslash \bar{K}_{n-1}\right)}{\operatorname{deg}\left(K_{n}^{-} / K_{n-1}\right)} \geq \frac{\bmod \left(K_{n-1}^{\prime} \backslash \bar{K}_{n-1}\right)}{C_{\mathcal{A}}^{2} C_{\Gamma}^{\tau}}, \\
\left.\bmod \left(K_{n+1} \backslash \overline{\mathcal{L}_{x}\left(K_{n+1}\right.}\right)\right) & \geq \bmod \left(K_{n+1} \backslash \overline{Q_{n+1}}\right) \\
& \stackrel{\text { vacuous }}{=} \frac{\bmod \left(K_{n} \backslash \overline{K_{n}^{-}}\right)}{\operatorname{deg}\left(K_{n+1} / K_{n}\right)} \geq \frac{\bmod \left(K_{n} \backslash \overline{K_{n}^{-}}\right)}{C_{K}} .
\end{aligned}
$$

Now Theorem 2.1 proves that $\bmod \left(K_{n}^{\prime} \backslash \bar{K}_{n}\right)$ for all $n$ have a positive lower bound, depending only on $b, \delta$, and $\widehat{\mu}$. It follows that $\bmod \left(K_{n} \backslash \overline{K_{n}^{-}}\right)$and $\bmod \left(K_{n} \backslash \overline{\mathcal{L}_{y}\left(K_{n}\right)}\right)$ for any $n$ and any $y \in \widehat{\mathcal{P}}_{f} \cap K_{n}$ have also a positive lower bound depending only on $b, \delta$, and $\widehat{\mu}$.

Notice that (27) is a crucial point for proving rigidity and non-invariant-line-field properties of $K_{f}$; see for example [KSS, $\mathrm{KS}, \mathrm{YZ}$.

To our best knowledge the inequality (28) appears here for the first time.

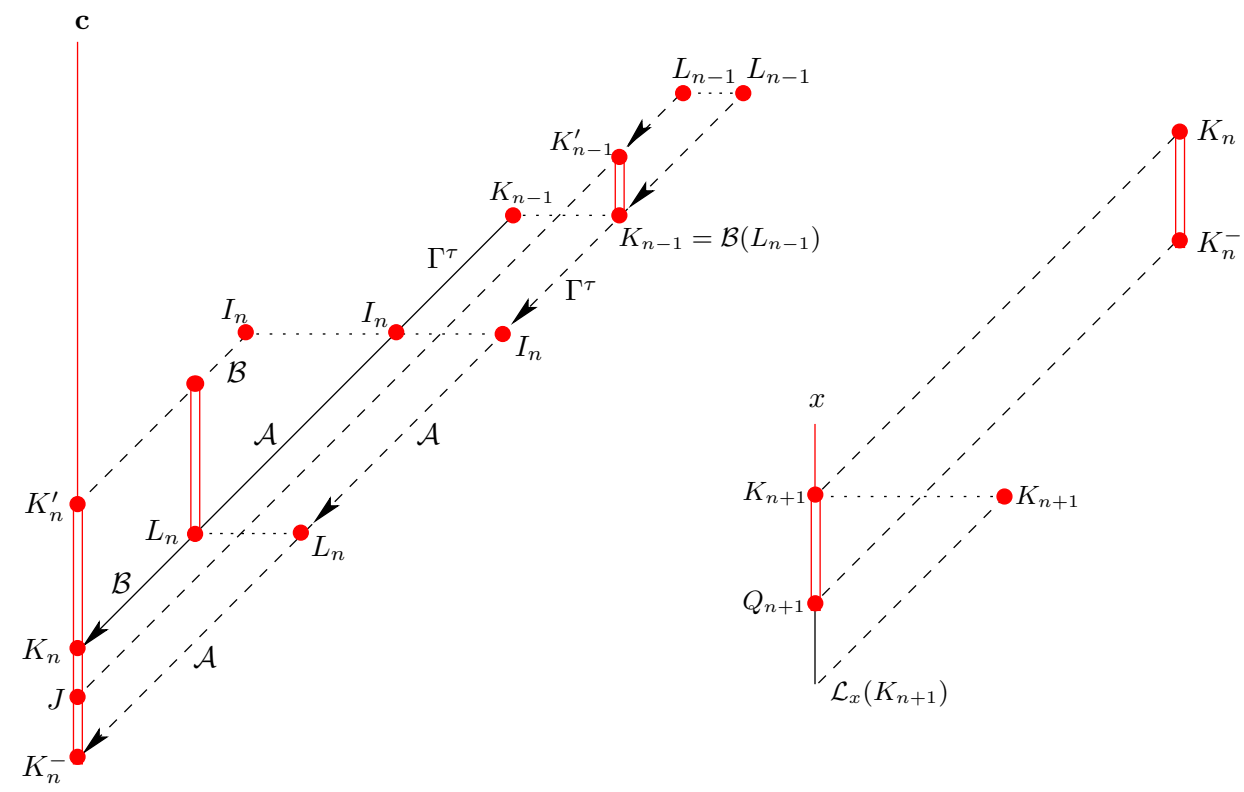

Figure 7. Control of moduli 


\section{Appendix A. Existence of AT LEASt two suCCEssors}

Proof of Lemma 4.2. (a) By the hypothesis of recurrence, $J$ has at most finitely many successors. We only need to show that $J$ has at least two successors.

See Figure 8 for the following construction.

On $\mathcal{T}(\mathbf{c})$, row $(J)$, denote by $k_{0}=0<k_{1}<k_{2}<\cdots$ the column numbers of the successive red spots. By the hypothesis of recurrence, this sequence of $k_{j}$ exists and is infinite.

Set $Q=\mathcal{L}_{\mathbf{c}}(J)$ to be the critical piece which is the pullback of the first $\geq 1$ visit of $\mathbf{c}$ to $J$. So $\left|Q /^{J}\right|=k_{1}$.

Clearly $Q$ is the first successor of $J$. We will prove:

Claim 1. Assume that for some $j \geq 1$ the red vertical segment on column $k_{j}$ does not reach $\operatorname{row}(Q)$. Then $J$ has a second successor.

Proof. Let us start from the row of $J$ at column $k_{j+1}$ and follow the left down diagonal until we reach a piece, say named by $Q^{\prime}$, at $k_{j}$-column. Then $Q^{\prime}$ is not critical, for otherwise $\operatorname{depth}\left(Q^{\prime}\right)<\operatorname{depth}(Q)$ by Rule 1 and the choice of $j$, and then we can apply Rule 2 and the definition of $k_{1}$ to find a contradiction.

Applying Lemma 4.1 we know that the closed diagonal segment $\left[Q^{\prime} /{ }^{J}\right]$ meets every critical color at most once.

Continue to follow that diagonal from $Q^{\prime}$ left downwards until we reach a critical vertex $W$. If $W$ is at the 0 column we are done. Otherwise let $S$ be the pullback of the first $\geq 0$ hit of $\mathbf{c}$ to $W$. Then $S$ is a successor of $J$. It must be deeper than $Q$ for otherwise moving the triangle with edge $\left.{ }_{[S} /^{J}\right]$ to the 0 -th column one gets a contradiction with the choice of $k_{1}$ and Rule 2 (the same argument also proves that $S$ must be on the 0 -th column, had we chosen $j$ minimal).

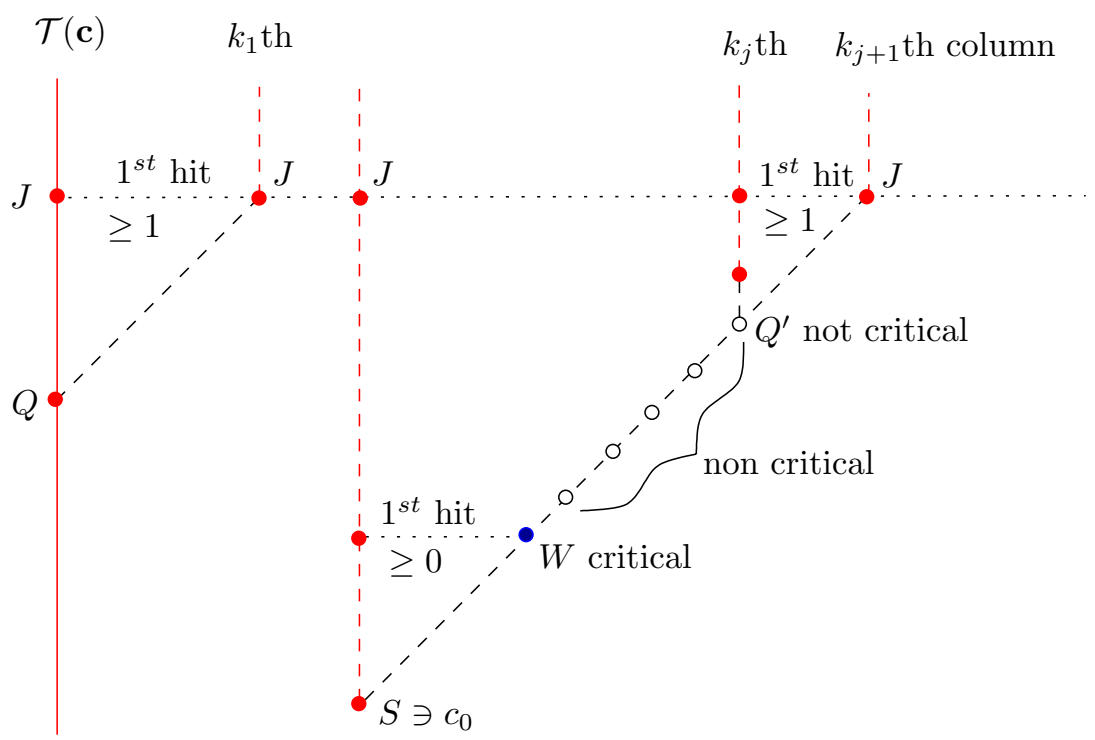

Figure 8. A second successor 
Claim 2. There is $j \geq 1$ such that the red vertical segment at column $k_{j}$ does not reach the row of $Q$.

Proof. Assume the contrary. Then by induction and Rule 2 one sees the following repeated pattern of identical upper triangles:

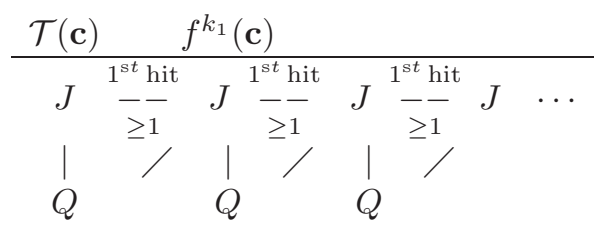

Therefore $k_{j}=j \cdot k_{1}$.

Denote by $n_{0}$ the depth of $J$. Then $Q$ is at depth $n_{0}+k_{1}$. Now consider the row at depth $n_{0}+2 k_{1}$. Denote its 0 -column vertex by $R$ and $k_{1}$-column vertex by $R^{\prime}$. As $R$ is critical, it is the pullback of the first $\geq 1$ hit of $\mathbf{c}$ to $Q$ :

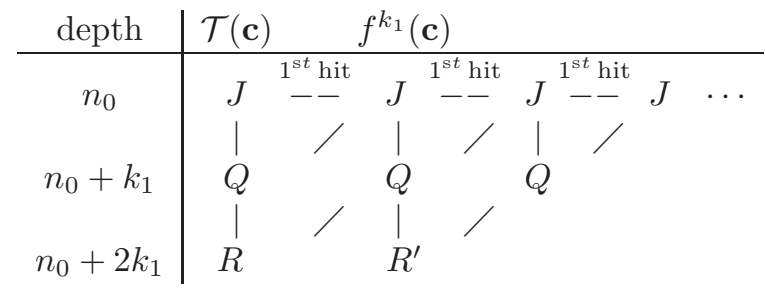

We will show now that $R^{\prime}$ is red.

Assume that for some $0<p<k_{1}, f^{p}(Q)$ is critical, say of color green (it cannot be red by Lemma 4.11). Then it follows by Lemma 4.1 Rules 1 and 2, that the green vertices on $\operatorname{row}\left(f^{p}(Q)\right)$ are precisely at the columns: $p+j \cdot k_{1}, j=0,1, \cdots$.

Consequently, by Rule 1, on any row deeper than $\operatorname{row}\left(f^{p}(Q)\right)$, the green vertices are contained in the columns $p+j \cdot k_{1}, j=0,1, \cdots$.

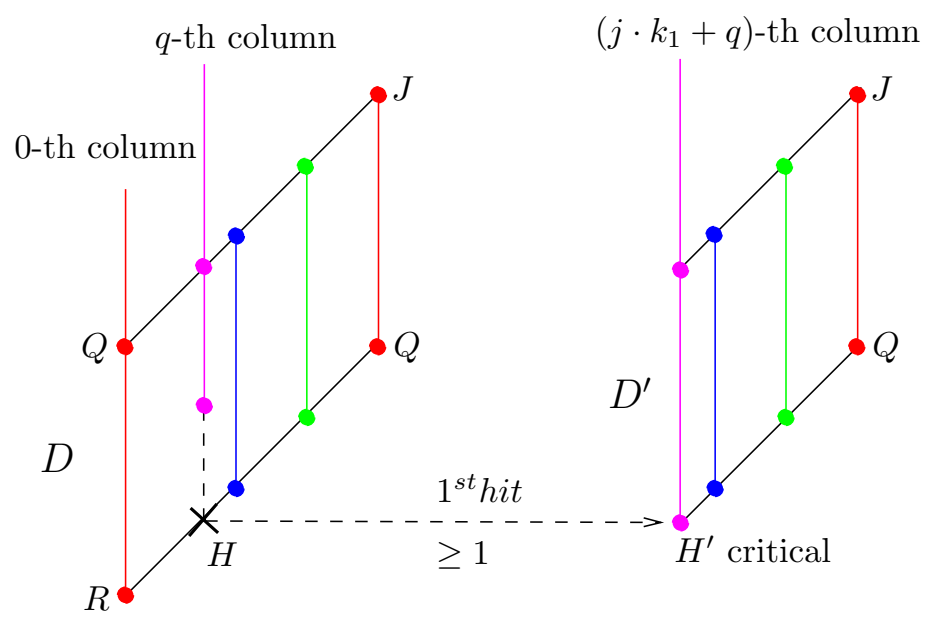

Figure 9. Critically full parallelograms 
Denote by $D$ the vertical parallelogram with edges ${\underset{R}{Q}}_{l^{2}}$ and ${ }_{Q}^{J}$, on the 0 -th and $k_{1}$-th column respectively. We will show that $D$ is critically full by induction from right to left on the consecutive critical vertical segments. (See Figure 9)

Assume that on $]_{R} / Q$ [, a piece $H$ is unknown to be critical or not, but is on the column of a critical piece that touches $1 /^{J}$ [, say of color pink, and that every critical vertical segment on the right of $\operatorname{column}(H)$ either does not touch $D$ or crosses through $D$. Denote by $q$ the column number of $H$.

By the hypothesis of recurrence, one can march horizontally from $H$ to the right, some positive number of steps until the first hit of a pink vertex $H^{\prime}$. Then $H^{\prime}$ is on the column $j \cdot k_{1}+q$ for some $j$. And the parallelogram $D^{\prime}$ with vertices $H^{\prime}, Q, J$ (with $Q, J$ on the $(j+1) k_{1}$-th column) is critically full: for its left edge is entirely critical by applying Rule 1 to $H^{\prime}$, and any of its other critical vertical segments will certainly reach the lower edge by applying Rule 3 to the induction assumption on $D$. Apply Rule 3 again, but this time from $D^{\prime}$ to $D$, we conclude that $H$ is also critical.

This ends the induction step and proves that $D$ is critically full. We then use it and Rule 3 to prove that the parallelogram of the same size as $D$, but with $R^{\prime}$ as the lower left vertex, is identical to $D$. In particular $R^{\prime}$ is critical.

Continuing this process one shows that the entire $k_{1}$-column is critical. This is impossible by the hypothesis of recurrence and ends the proof of Claim 2.

This proves (a).

Part (b). Let $S$ be a successor of $J$. From the definition we know that the half open diagonal segment ${ }_{[S} /^{J}[$ meets at most $2 b-1$ critical pieces, and only one red spot (i.e. $S)$. Therefore $\left\{\begin{array}{l}\operatorname{deg}\left(S^{J}\right) \leq \delta^{2 b-1} ; \\ \#\left(\{\text { red spots }\} \cap{ }_{[S} /{ }^{J}[)=1 .\right.\end{array}\right.$ This implies Formula (66) with $S$ replaced by $\Gamma(J)$.

\section{Appendix B. Preparations for the operators $\mathcal{A}$ and $\mathcal{B}$}

The idea is to take special pullbacks of some critical puzzle pieces in order to decrease their sizes to get a better control of locations of the postcritical set.

One easily verifies that this construction still holds in the more general setup where the domain $\mathbf{U}$ of $f$ is only included in the range $\mathbf{V}$ (but not necessarily compactly contained in) (see RY] ).

We will switch the notation of the red colored critical point from $\mathbf{c}$ to $c_{0}$, and use the letter $c$ to denote an arbitrary critical point.

Proposition B.1. Fix a critical point $c_{0} \in$ Crit $(f)$. Color it red. Fix a critical puzzle piece $I$ containing $c_{0}$. Then there is a collection of pairs of critical puzzle pieces

$$
\left\{P_{c} \ni P_{c}^{\prime} \ni c \mid c \in \operatorname{Crit}(f)\right\}
$$

satisfying: for any $c \in C r i t(f)$, both $P_{c}$ and $P_{c}^{\prime}$ are pullbacks of $I$, and

$$
\left\{\begin{array}{l}
\operatorname{deg}\left(P_{c} /^{I}\right) \leq \delta^{b^{2}-b} \\
\#\left(\{\text { red spots }\} \cap{ }_{\left[P_{c}\right.} /^{I}[) \leq b-1\left(P_{c}=I \text { if } b=1\right) .\right.
\end{array}\right.
$$

Furthermore, if a postcritical point $x$ appears in some annulus $P_{c} \backslash \overline{P_{c}^{\prime}}$, then there is a puzzle piece $V$ containing $x$, such that $V$ and $P_{c}^{\prime}$ are two disjoint puzzle pieces 
within $P_{c}$, and that for some integer $k, f^{k}$ maps $V$ conformally onto $P_{\hat{c}}$ for some $\hat{c} \in \operatorname{Crit}(f)$.

The construction and the properties of $P_{c}, P_{c}^{\prime}$ are given in [KSS, pp. 802-805], (but without all the details of the proof). We give a slightly different presentation, together with a complete proof.

At first, some notations:

We say that a puzzle piece $V$ is nested (respectively strictly nested) in another puzzle piece $U$ if one of the following (equivalent) events happens:

1. $V \subset U$ (respectively $V \Subset U)$;

2. in some tableau $V$ and $U$ appear in the same column with $V$ below or equal to $U$ (respectively with $V$ below $U$ );

3. $\partial V \cap \bar{U} \neq \emptyset$ (respectively $\partial V \cap U \neq \emptyset$ ).

Let $\widehat{H}=\bigcup_{i \in \Lambda} U_{i}$ be a union of finitely many disjoint puzzle pieces so that each $U_{i}$ contains a unique critical point $c_{i}$ (the $U_{i}$ 's do not necessarily have the same depth). An $\widehat{H}$-piece will be one of $U_{i}$. An $\widehat{H}$-subpiece will be a puzzle piece $W$ nested in an $\widehat{H}$-piece. We say that $\widehat{H}$ is strictly nice, if for any $n>0, f^{n}(\partial \widehat{H}) \cap \operatorname{closure}(\widehat{H})=\emptyset$. This is equivalent to say that for any $i \in \Lambda$, any upper triangle of any size with lower vertex $U_{i}$ contains no other entry (except that of $U_{i}$ ) that is an $\widehat{H}$-piece. This is also equivalent to say that for any $i \in \Lambda$, any upper triangle of any size with lower vertex $U_{i}$ contains no other entry (except that of $U_{i}$ ) that is an $\widehat{H}$-subpiece.

We extend also the notion of $\mathcal{L}_{x}(U)$ to $\mathcal{L}_{x}(\widehat{H})$. Let $x \in \mathbb{C}$. We define $\mathcal{L}_{x}(\widehat{H})$, the pullback of the first $\geq 1$ hit of $x$ to $\widehat{H}$ as follows: if for any $l>0, f^{l}(x) \notin \widehat{H}$, set $\mathcal{L}_{x}(\widehat{H})=\emptyset$; if there is $l>0$ minimal so that $f^{l}(x) \in \widehat{H}$, then $f^{l}(x) \in U_{q}$ for some $q \in \Lambda$, set $\mathcal{L}_{x}(\widehat{H}):=\mathcal{L}_{x}\left(U_{q}\right)$. Here is an equivalent definition. For each $i \in \Lambda$ start at the 0 -th column of $\mathcal{T}(x)$, row $\left(U_{i}\right)$, march right $l_{i} \geq 1$ steps until the first hit of $U_{i}$ (if any, otherwise set $l_{i}=+\infty$ ). Then there is a unique $q$ so that $l_{q}$ is minimal among all the $l_{i}$ 's (for if $l_{j}=l_{q}$, then $U_{j}$ and $U_{q}$ appear both on the $l_{j}$-th column of $\mathcal{T}(x)$ but with different depths, resulting that one of them is nested in the other). Then $\mathcal{L}_{x}(\widehat{H}):=\mathcal{L}_{x}\left(U_{q}\right)$ is the pullback piece by $f^{l_{q}}$ of $U_{q}$ containing $x$.

In case $\widehat{H}$ is strictly nice, we may locate $\mathcal{L}_{x}(\widehat{H})$ in a more precise way. Let $U_{i}$ be one of the $\widehat{H}$-pieces of the least depth such that $f^{k}(x) \in U_{i}$ for some $k>0$. Start from the 0 -th column of $\mathcal{T}(x)$ at $\operatorname{row}\left(U_{i}\right)$. March $l>0$ steps to the right until the first hit of $U_{i}$. Follow its left-down diagonal until the 0 -th column, to reach the piece $\mathcal{L}_{x}\left(U_{i}\right)$. Denote by $S$ the upper triangle with lower vertex $\mathcal{L}_{x}\left(U_{i}\right)$ and right vertex $U_{i}$. Among the $\widehat{H}$-pieces appearing in $S$ (except those on the 0 -th column), choose $U_{q}$ that has the least column number. Then $\mathcal{L}_{x}(\widehat{H})$ is the piece on the vertical edge of $S$ that is on the diagonal of $U_{q}$.

We define also $J_{\widehat{H}}:=\bigcup_{i \in \Lambda} \mathcal{L}_{c_{i}}(\widehat{H})=\bigcup_{i \in \Lambda} \mathcal{L}_{c_{i}}\left(U_{q_{i}}\right)$ for some suitable index $q_{i} \in \Lambda$.

Similarly one can define $L_{x}^{2}(\widehat{H})$, the pullback of the second hit of $x$ to $\widehat{H}$, to be the pullback piece by $f^{k}$ of the $\widehat{H}$-piece containing $f^{k}(x)$, in the case that $k>l>0$ are the two minimal integers such that $f^{l}(x), f^{k}(x) \in \widehat{H}\left(\operatorname{set} L_{x}^{2}(\widehat{H})=\emptyset\right.$ if such $k$ does not exist). Notice that $L_{x}^{2}(\widehat{H})$ is not necessarily $\mathcal{L}_{x}\left(\mathcal{L}_{x}(\widehat{H})\right)$.

The key in the proof of this proposition is the following lemma.

Lemma B.2. Let $\widehat{H}=\bigcup_{i \in \Lambda} U_{i}$, such that each $U_{i}$ is a puzzle piece containing a unique critical point $c_{i}$, that $U_{i}$ and $U_{j}$ have disjoint closures when $i \neq j$, and that 
$\widehat{H}$ is strictly nice. Numerate the set of critical points into $\left\{c_{0}, \cdots, c_{b-1}\right\}$ so that $\Lambda=\{0,1, \cdots, s-1\}$ for some $0<s \leq b$. Then

(a) $J_{\widehat{H}} \Subset \widehat{H}$.

(b) Assume that there is a critical point c outside $\widehat{H}$ such that $f^{l}(c) \in \widehat{H} \backslash \overline{J_{\widehat{H}}}$ for the minimal $l>0$ satisfying $f^{l}(c) \in \widehat{H}$. By the hypothesis of recurrence there is $k>l$ minimal such that $f^{k}(c) \in \widehat{H}$. Then

(b1) for the non-empty puzzle piece $L_{c}^{2}(\widehat{H})$, we have $f^{l}\left(L_{c}^{2}(\widehat{H})\right) \subset \widehat{H} \backslash \overline{J_{\widehat{H}}}$ and, for $0 \leq j<k$ and $j \neq l$, the $j$-th column of $\mathcal{T}(c)$ does not contain any $\widehat{H}$-subpieces, and $\operatorname{deg}\left(\left.f^{k}\right|_{L_{c}^{2}(\widehat{H})}\right) \leq \delta^{2(b-s)}$;

(b2) for each $i \in\{0,1, \cdots, s-1\}$, denote by $l_{i}>0$ the unique integer such that $f^{l_{i}}\left(\mathcal{L}_{c_{i}}(\widehat{H})\right)$ is an $\widehat{H}$-piece, then $\operatorname{deg}\left(\left.f^{l_{i}}\right|_{\mathcal{L}_{c_{i}}(\widehat{H})}\right) \leq \delta^{b-s+1} \leq \delta^{2(b-s)}$;

(b3) the set $\widehat{H}^{\prime}:=J_{\widehat{H}} \cup L_{c}^{2}(\widehat{H})$ is strictly nice.

(c) Denote by $\mathcal{C}=\left\{c_{s}, \cdots, c_{b-1}\right\}$ the set of critical points outside $\widehat{H}$ (we allow the case that $\mathcal{C}=\emptyset$ ). Assume, contrary to the point (b) above, that the orbit of every $c \in \mathcal{C}$ (if any) enters $J_{\widehat{H}}$ when it enters $\widehat{H}$ for the first time. Set $P_{c}=\mathcal{L}_{c}(\widehat{H})$ and $P_{c}^{\prime}=\mathcal{L}_{c}\left(J_{\widehat{H}}\right)$ for any $c \in \mathcal{C}$. Then for any $x \in \widehat{\mathcal{P}}_{f} \cap\left(\left(\widehat{H} \backslash \overline{J_{\widehat{H}}}\right) \cup \bigcup_{c \in \mathcal{C}}\left(P_{c} \backslash \overline{P_{c}^{\prime}}\right)\right)$ (if any), there is a puzzle piece $V_{x}$ containing $x$ and an integer $k$ such that $f^{k}$ maps $V_{x}$ conformally onto a component of $\widehat{H} \cup \bigcup_{c \in \mathcal{C}} P_{c}=\bigcup_{i=0}^{s-1} U_{i} \cup \bigcup_{c \in \mathcal{C}} P_{c}$.

Proof. The following properties (A) and (B) of $\widehat{H}$ are essential as a strictly nice set:

(A) For any puzzle piece $V$ with an half open diagonal segment $]_{V} /^{*}$ ] containing an $\widehat{H}$-piece (i.e. some iterate of $V$ is an $\widehat{H}$-piece), then no $\widehat{H}$-piece is nested in $V$, and no piece of the form $\mathcal{L}_{x}(\widehat{H})$ is strictly nested in $V$. Furthermore, if for a point $y$, there are two minimal integers $k>l>0$ such that $f^{l}(y), f^{k}(y) \in \widehat{H}$, then, setting $z=f^{l}(y)$, we have that $\mathcal{L}_{z}(\widehat{H})$ is strictly nested in an $\widehat{H}$-piece and $L_{y}^{2}(\widehat{H})$ is strictly nested in $\mathcal{L}_{y}(\widehat{H})$.

\begin{tabular}{l}
$y \quad z=f^{l}(y)$ \\
\hline$\times{ }^{1^{s t} \text { hit }} U_{p} \quad U_{q}=f^{k}\left(L_{y}^{2}(\widehat{H})\right)$ \\
$\mid \mathcal{L}_{y}(\widehat{H}) \stackrel{\mathcal{L}_{z}(\widehat{H})}{\mid}$ \\
$L_{y}^{2}(\widehat{H}) \quad$ (here $\operatorname{row}\left(U_{p}\right)$ may or may not equal to $\left.\operatorname{row}\left(U_{q}\right)\right)$.
\end{tabular}

Proof. If $V \supset U_{i}$ for some $\widehat{H}$-piece $U_{i}$, then an upper triangle with lower vertex $U_{i}$ would contain the $\widehat{H}$-piece in the diagonal starting from $V$. This contradicts that $\widehat{H}$ is strictly nice.

Assume that $\mathcal{L}_{x}(\widehat{H})$ for some $x$ is strictly nested in $V$. Let $l, m$ be the two $>0$ integers such that $f^{l}(V), f^{m}\left(\mathcal{L}_{x}(\widehat{H})\right)$ are two $\widehat{H}$-pieces. As $\widehat{H}$-pieces are not nested into each other, $l \neq m$. But $l<m$ would contradict that $\mathcal{L}_{x}(\widehat{H})$ is the pullback of the first hit of $x$ to $\widehat{H}$, and $l>m$ would contradict that $\widehat{H}$ is strictly nice (by looking at an upper triangle with lower vertex $\left.f^{m}\left(\mathcal{L}_{x}(\widehat{H})\right)\right)$. 
Now for a point $y$ and for $z=f^{l}(y)$ its first visit to $\widehat{H}$, say $z \in U_{p}$. Then by above $\mathcal{L}_{z}(\widehat{H})$ is strictly nested in $U_{p}$. It follows that $L_{y}^{2}(\widehat{H})$ is strictly nested in $\mathcal{L}_{y}(\widehat{H})$.

(B) For $z$ in the following three cases: $\left\{\begin{array}{l}(1)=c_{i}, i \in \Lambda \\ (2) \notin \widehat{H} \\ (3) \in \widehat{H} \backslash \overline{J_{\widehat{H}}},\end{array}\right.$ such that there is $l>0$ minimal with $f^{l}(z) \in \widehat{H}$, then for any $\left\{\begin{array}{l}(1) 0<j<l \\ (2) 0 \leq j<l \\ (3) 0<j<l,\end{array}\right.$ the $j$-th column of $\mathcal{T}(z)$ contains no $\widehat{H}$-(sub)pieces; $\operatorname{deg}\left(\left.f^{l}\right|_{\mathcal{L}_{z}(\widehat{H})}\right) \leq\left\{\begin{array}{ll}\delta^{b-s+1} & \text { in case (1) } \\ \delta^{b-s} & \text { in cases (2) and (3) }\end{array} ;\right.$ and $\mathcal{L}_{z}(\widehat{H}) \subset \widehat{H} \backslash \overline{J_{\widehat{H}}}$ in case $(3)$.

Proof. The tableau interpretation of Case (2) (respectively (3)) is: the 0-th column of $\mathcal{T}(z)$ contains no $\widehat{H}$-(sub)pieces (respectively contains an $\widehat{H}$-piece but not a $J_{\widehat{H}^{-}}$ piece).

Let $z$ be in one of the three cases. Fix $0<j<l$ (if any). The puzzle piece $f^{j}\left(\mathcal{L}_{z}(\widehat{H})\right)$ is a pullback of $\widehat{H}$ containing a point (the point $\left.f^{j}(z)\right)$ not in $\widehat{H}$, so $f^{j}\left(\mathcal{L}_{z}(\widehat{H})\right)$ is not nested in any $\widehat{H}$-piece. On the other hand, by (A), no $\widehat{H}$-piece is nested in $f^{j}\left(\mathcal{L}_{z}(\widehat{H})\right)$. So the $j$-th column of $\mathcal{T}(z)$ does not contain any $\widehat{H}$-piece.

Now as the orbit of $z$ hits some $U_{q}$ for the first $\geq 1$ time, we know that the

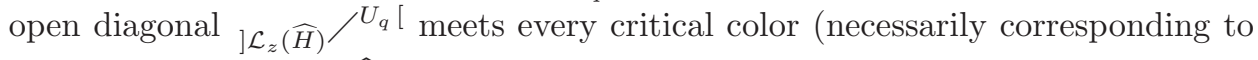
critical points outside $\widehat{H})$ at most once. So $\operatorname{deg}\left(\left.f^{l_{i}}\right|_{\mathcal{L}_{c_{i}}(\widehat{H})}\right) \leq \delta^{b-s}$ in the cases (2) and (3), and $\leq \delta^{b-s+1}$ in case (1).

Finally in case (3), there is an $i \in\{0, \cdots, s-1\}$ such that $z \in U_{i} \backslash \overline{\mathcal{L}_{c_{i}}(\widehat{H})}$. We apply (A) to conclude that $\mathcal{L}_{z}(\widehat{H}) \Subset U_{i}$ and $\mathcal{L}_{z}(\widehat{H}) \cap \mathcal{L}_{c_{i}}(\widehat{H})=\emptyset$. So $\mathcal{L}_{z}(\widehat{H}) \subset$ $\widehat{H} \backslash \overline{J_{\widehat{H}}}$.

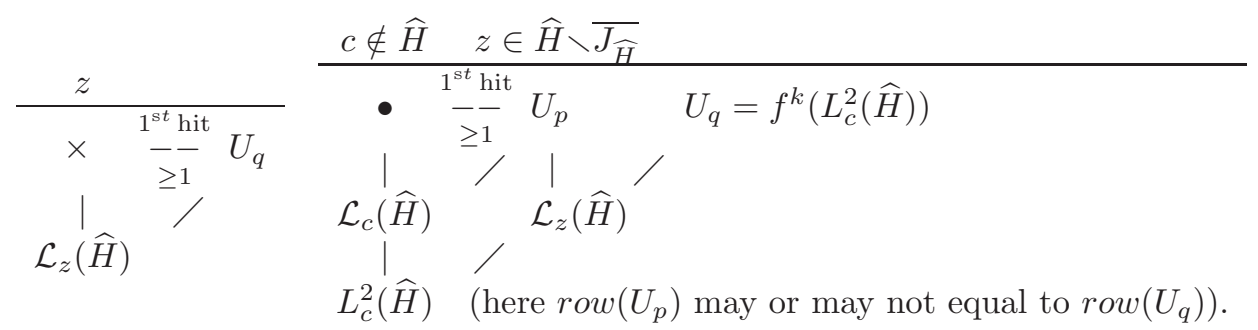

We start now the proof of our lemma.

(a) For each $j=0, \cdots, s-1$, the set $\mathcal{L}_{c_{j}}(\widehat{H})$ is a puzzle piece containing $c_{j}$ and is a pullback of an $\widehat{H}$-piece, so by (A) it is strictly nested in $U_{j}$, the $\widehat{H}$-piece containing $c_{j}$. So $J_{\widehat{H}} \Subset \widehat{H}$.

(b1) and (b2): We just need to apply once (B) case (3) to $z=f^{l}(c)$ and once (B) case (2) to $z=c$, and use the fact that $L_{c}^{2}(\widehat{H}) \Subset \mathcal{L}_{c}(\widehat{H})$. We get also $\operatorname{deg}\left(\left.f^{k}\right|_{L_{c}^{2}(\widehat{H})}\right) \leq \delta^{2(b-s)}$. Now the existence of $c$ outside $\widehat{H}$ implies that $b \geq s+1$. So $2(b-s) \geq b-s+1$. Therefore by (B) case (1) we have $\operatorname{deg}\left(\left.f^{l_{i}}\right|_{\mathcal{L}_{c_{i}}(\widehat{H})}\right) \leq \delta^{b-s+1} \leq$ $\delta^{2(b-s)}$ for $i=0, \cdots, s-1$.

(b3) Proof of the niceness of $\widehat{H}^{\prime}=J_{\widehat{H}} \cup L_{c}^{2}(\widehat{H})$. 
Consider at first $L_{c}^{2}(\widehat{H})$. Denote by $D$ the largest possible upper triangle with lower vertex $L_{c}^{2}(\widehat{H})$. Then any tableau entry in $D$, except the lowest vertex, will have strictly less depth than $\operatorname{depth}\left(L_{c}^{2}(\widehat{H})\right)$, thus cannot be equal to $L_{c}^{2}(\widehat{H})$.

We want to show that $D$ does not contain any $J_{\widehat{H}}$-piece neither. By (a) every $J_{\widehat{H}}$-piece is an $\widehat{H}$-subpiece. As $c \notin \widehat{H}$ the 0 -th column of $\mathcal{T}(c)$ will not contain any $\widehat{H}$-subpiece in particular not contain any $J_{\widehat{H}}$-piece. Let $k>l>0$ be the smallest integers such that $f^{k}(c), f^{l}(c) \in \widehat{H}$. We know from (b1) that for any $0<j<k$ and $j \neq l$, the $j$-th column of $\mathcal{T}(c)$ does not contain any $\widehat{H}$-subpiece, in particular not any $J_{\widehat{H}}$-piece. But the $l$-th column does not contain $J_{\widehat{H}}$-pieces neither, as $f^{l}\left(L_{c}^{2}(\widehat{H})\right) \subset \widehat{H} \backslash \overline{J_{\widehat{H}}}$ by (b1). Now $f^{k}\left(L_{c}^{2}(\widehat{H})\right)=U_{q}$ is an $\widehat{H}$-piece. Using now the fact that $\widehat{H}$ is nice to conclude that the sub-upper triangle of $D$ with lower vertex $U_{q}$ does not contain any other $\widehat{H}$-subpieces except $U_{q}$, consequently it does not contain any $J_{\widehat{H}}$-pieces.

Therefore $D$ does not contain $\widehat{H}^{\prime}$-pieces except at its lower vertex.

Consider now a $J_{\widehat{H}}$-piece which is $\mathcal{L}_{c_{i}}(\widehat{H})$ for some $0 \leq i \leq s-1$. Denote by $D_{i}$ the largest upper triangle with lower vertex $\mathcal{L}_{c_{i}}(\widehat{H})$. By similar argument as above one proves easily that $D_{i}$ does not contain any other $J_{\widehat{H}}$-pieces.

The piece $L_{c}^{2}(\widehat{H})$ does not appear on the 0 -th column of $\mathcal{T}\left(c_{i}\right)$ for if it appears above $U_{i}$ that would contradict the niceness of $\widehat{H}$, and if it appears below or equal to $U_{i}$, that would contradict that $U_{i}$ contains no critical point other than $c_{i}$.

Assume by contradiction that there is some $n>0$ such that $L_{c}^{2}(\widehat{H})$ appears in the $n$-th vertical segment of $D_{i}$. Then $\mathcal{L}_{c}(\widehat{H})$ appears above $L_{c}^{2}(\widehat{H})$ on the same column. Follow now the left-down diagonal from $\mathcal{L}_{c}(\widehat{H})$ to the 0 -th column, we will find a piece $V$ that is a pullback of an $\widehat{H}$-piece, and is above $\mathcal{L}_{c_{i}}(\widehat{H})$. This contradicts (A).

This means that $\widehat{H}^{\prime}$ is strictly nice.

(c) Set $\widetilde{H}=\widehat{H} \cup \bigcup_{c \in \mathcal{C}} P_{c}$.

Consider at first the case $x \in \widehat{\mathcal{P}}_{f} \cap\left(\widehat{H} \backslash \overline{J_{\widehat{H}}}\right)$. Set $V_{x}=\mathcal{L}_{x}(\widetilde{H})$. Let $k>0$ be the minimal integer such that $f^{k}(x) \in \widetilde{H}$ (such $k$ exists by recurrence assumption). Then $f^{k}\left(V_{x}\right)$ is an $\widetilde{H}$-piece.

By (A) we have $V_{x} \subset U_{i} \backslash \overline{\mathcal{L}_{c_{i}}(\widehat{H})}$ for some $i \in\{0, \cdots, s-1\}$. So $V_{x}$ does not contain any critical point. On the other hand, since all the critical points are

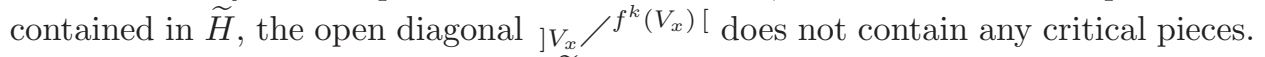
So $f^{k}$ maps $V_{x}$ univalently onto an $\widetilde{H}$-piece.

Consider now $x \in \widehat{\mathcal{P}}_{f} \cap\left(P_{c} \backslash \overline{P_{c}^{\prime}}\right)$ for some $c \in \mathcal{C}$. We define $V_{x}$ in three steps:

- let $y=f^{l_{x}}(x)$ be the first point in the orbit of $x$ that visits $\widehat{H}$ (such $y$ exists by recurrence assumption);

- set $V_{y}=\mathcal{L}_{y}(\widetilde{H})$

- finally define $V_{x}$ to be the pullback by $f^{l_{x}}$ of $V_{y}$ containing $x$.

If $f^{j}\left(P_{c}\right)$ contains a critical point $\tilde{c}$ for some $0<j<l_{x}$, then $f^{j}\left(P_{c}\right)=P_{\tilde{c}}$ and $f^{j}\left(P_{c}^{\prime}\right)=P_{\tilde{c}}^{\prime}$ by $(\mathrm{A})$ and the fact that $f^{l_{x}}(c) \in J_{\widehat{H}}$. It follows that $f^{l_{x}}$ acts as a covering from the annulus $P_{c} \backslash \overline{P_{c}^{\prime}}$ onto the annulus $U_{i} \backslash \overline{\mathcal{L}_{c_{i}}(\widehat{H})}$ for some $0 \leq$ $i \leq s-1$. This implies that $y \in \widehat{\mathcal{P}}_{f} \cap\left(U_{i} \backslash \overline{\mathcal{L}_{c_{i}}(\widehat{H})}\right) \subset \widehat{\mathcal{P}}_{f} \cap\left(\widehat{H} \backslash \overline{J_{\widehat{H}}}\right)$. By above 
there is an integer $k_{y}>0$ such that $f^{k_{y}}$ maps $V_{y}$ univalently onto an $\widetilde{H}$-piece, and $V_{y} \subset U_{i} \backslash \overline{\mathcal{L}_{c_{i}}(\widehat{H})}$. But any covering over a simply connected domain is trivial. So $f^{l_{x}}$ maps $V_{x}$ univalently onto $V_{y}$. This implies that $f^{l_{x}+k_{y}}$ maps $V_{x}$ univalently onto an $\widetilde{H}$-piece.

Proof of Proposition B.1. Recall that $I$ is a puzzle piece containing a critical point named by $c_{0}$, which is colored red.

Step 1. We will define at first

- an integer $m$ with $0 \leq m<b$;

- a re-numeration of the critical points into $c_{0}, c_{1}, \cdots, c_{m} ; c_{m+1}, \cdots, c_{b-1}$; and

- an array of puzzle pieces $p_{l i}$ of $c_{i}$ for $i \leq m, i \leq l \leq m+1$ as follows:

\begin{tabular}{c|llllll} 
& red & & & & & \\
& $c_{0}$ & $c_{1}$ & $c_{2}$ & $c_{3}$ & $\cdots$ & $c_{m}$ \\
\hline$\widehat{H}_{0}$ & $p_{00}=I$ & & & & & \\
$\widehat{H}_{1}$ & $p_{10}$ & $p_{11}$ & & & & \\
$\widehat{H}_{2}$ & $p_{20}$ & $p_{21}$ & $p_{22}$ & & & \\
$\widehat{H}_{3}$ & $p_{30}$ & $p_{31}$ & $p_{32}$ & $p_{33}$ & & \\
$\vdots$ & $\vdots$ & $\vdots$ & $\vdots$ & $\vdots$ & $\cdots$ & \\
$\widehat{H}_{m}$ & $p_{m 0}$ & $p_{m 1}$ & $p_{m 2}$ & $p_{m 3}$ & $\cdots$ & $p_{m m}$ \\
$J_{m}$ & $p_{(m+1) 0}$ & $p_{(m+1) 1}$ & $p_{(m+1) 2}$ & $p_{(m+1) 3}$ & $\cdots$ & $p_{(m+1) m}$
\end{tabular}

We endow the set of critical puzzle pieces of a same critical point their natural ordering: $P \leq Q$ if $P \subset Q$.

O. Set $p_{00}=I$ and $p_{10}=\mathcal{L}_{c_{0}}(I)$. Set $\widehat{H}_{0}=p_{00}, J_{0}=\mathcal{L}_{c_{0}}\left(\widehat{H}_{0}\right)$. We have $J_{0} \Subset \widehat{H}_{0}$, and that $\widehat{H}_{0}$ is strictly nice.

If for any $c \neq c_{0}$, its first visit to $\widehat{H}_{0}$ enters also $J_{0}$, set $m=0$, enumerate the remaining critical points randomly. Stop.

I. If not, choose $c_{1} \neq c_{0}$ whose first visit to $\widehat{H}_{0}$ is in $\widehat{H}_{0} \backslash \bar{J}_{0}$. Set $p_{11}=L_{c_{1}}^{2}\left(\widehat{H}_{0}\right)$. Set $\widehat{H}_{1}=p_{10} \cup p_{11}$. By Lemma B.2, the set $\widehat{H}_{1}$ is strictly nice and

$$
\begin{aligned}
& \left\{\begin{array}{l}
\operatorname{deg}\left(p_{11} / I\right) \leq \alpha \times \alpha=\delta^{2(b-1)} ; \\
\#\left(\{ \text { red spots } \} \cap \left[p_{11} / p_{00}[)=0,\right.\right.
\end{array}\right. \\
& \left\{\begin{array}{l}
\operatorname{deg}\left(p_{10} /{ }^{I}\right) \leq \delta^{b} ; \\
\#\left(\{\text { red spots }\} \cap{ }_{\left[p_{10}\right.} / p_{00}[)=1 .\right.
\end{array}\right.
\end{aligned}
$$

Set $p_{20}=\mathcal{L}_{c_{0}}\left(\widehat{H}_{1}\right)$ (it is one of $\left.\mathcal{L}_{c_{0}}\left(p_{10}\right), \mathcal{L}_{c_{0}}\left(p_{11}\right)\right)$ and $p_{21}=\mathcal{L}_{c_{1}}\left(\widehat{H}_{1}\right)$. Set finally $J_{1}=p_{20} \cup p_{21}$. By Lemma B.2 again, $J_{1} \Subset \widehat{H}_{1}$.

If for any $c \neq c_{0}, c_{1}$, its first visit to $\widehat{H}_{1}$ enters also $J_{1}$, set $m=1$, numerate the remaining critical points randomly. Stop.

II. If not, choose $c_{2} \neq c_{0}, c_{1}$ whose first visit to $\widehat{H}_{1}$ is in $\widehat{H}_{1} \backslash \bar{J}_{1}$. Set $p_{22}=$ $L_{c_{2}}^{2}\left(\widehat{H}_{1}\right)$. Set $\widehat{H}_{2}=p_{20} \cup p_{21} \cup p_{22}, p_{3 i}=\mathcal{L}_{c_{i}}\left(\widehat{H}_{2}\right), i=0,1,2$, and $J_{2}=p_{30} \cup p_{31} \cup p_{32}$. Apply Lemma B.2 to conclude that $\widehat{H}_{2}$ is strictly nice, $J_{2} \Subset \widehat{H}_{2}$, and

$$
\left\{\begin{array}{l}
\operatorname{deg}\left(p_{22} / \widehat{H}_{1}\right) \leq \delta^{2(b-2)} ; \\
\#\left(\{\text { red spots }\} \cap{ }_{\left[p_{22}\right.} / \operatorname{comp}\left(\widehat{H}_{1}\right)[]=0 ;\right.
\end{array}\right.
$$


and, for $i=0,1$,

$$
\left\{\begin{array}{l}
\operatorname{deg}\left(p_{2 i} \widehat{T}^{\widehat{H}_{1}}\right) \leq \delta^{b-1} \\
\#\left(\{\text { red spots }\} \cap{ }_{\left[p_{2 i}\right.} / \operatorname{comp}\left(\widehat{H}_{1}\right)[) \leq 1 .\right.
\end{array}\right.
$$

III. Continuing this process, until we reach an $m<b$, such that, $\widehat{H}_{m}:=p_{m 0} \cup$ $\cdots \cup p_{m m}$ is strictly nice, and for any $c \neq c_{0}, c_{1}, \cdots, c_{m}$ (if any), its first visit to $\widehat{H}_{m}$ enters also $J_{m}:=p_{(m+1) 0} \cup \cdots \cup p_{(m+1) m}$. Numerate the remaining critical points randomly. Stop.

By Lemma B.2, we have for any $i \leq m, J_{i} \Subset \widehat{H}_{i}$ and

$$
\left\{\begin{array}{l}
\operatorname{deg}\left(p_{i i} / \widehat{H}^{-1}\right) \leq \delta^{2(b-i)}, \\
\#\left(\{\text { red spots }\} \cap\left[p_{i i} /{ }^{\operatorname{comp} p\left(\widehat{H}_{i-1}\right)[}\right)=0 .\right.
\end{array}\right.
$$

And, for $j<i \leq m$,

$$
\left\{\begin{array}{l}
\operatorname{deg}\left(p_{i j} / \widehat{H}_{i-1}\right) \leq \delta^{b-i+1} \\
\#\left(\{\text { red spots }\} \cap{ }_{\left[p_{i j}\right.} / \operatorname{comp}\left(\widehat{H}_{i-1}\right)[) \leq 1 .\right.
\end{array}\right.
$$

Step 2. Now we define $P_{c_{i}}, P_{c_{i}}^{\prime}$ for every $i \in\{0, \cdots, d-1\}$. For $i \leq m$, set $P_{c_{i}}=p_{m i}, P_{c_{i}}^{\prime}=p_{(m+1) i}$. For $c_{j}$ with $j>m$ (if any), set $P_{c_{j}}=\mathcal{L}_{c_{j}}\left(\widehat{H}_{m}\right)$ and $P_{c_{j}}^{\prime}=\mathcal{L}_{c_{j}}\left(J_{m}\right)$. By Lemma B.2 $\operatorname{deg}\left(P_{c_{j}} /^{\widehat{H}_{m}}\right) \leq \delta^{b-(m+1)} \leq \delta^{2(b-(m+1))}$.

\begin{tabular}{r|lllllll}
$c$ & $c_{0}$ & $c_{1}$ & $c_{2}$ & $c_{3}$ & $\cdots$ & $c_{m}$ & $c_{j}(j>m)$ \\
\hline$P_{c}$ & $p_{m 0}$ & $p_{m 1}$ & $p_{m 2}$ & $p_{m 3}$ & $\cdots$ & $p_{m m}$ & $\mathcal{L}_{c_{j}}\left(\widehat{H}_{m}\right)$ \\
$P_{c}^{\prime}$ & $p_{(m+1) 0}$ & $p_{(m+1) 1}$ & $p_{(m+1) 2}$ & $p_{(m+1) 3}$ & $\cdots$ & $p_{(m+1) m}$ & $\mathcal{L}_{c_{j}}\left(J_{m}\right)$
\end{tabular}

Clearly every $P_{c}$ is mapped eventually onto $p_{00}=I$. And, by Lemma B.2.

$$
\left\{\begin{array}{l}
\operatorname{deg}\left(P_{c} /^{I}\right) \leq \delta^{2(b-1)+2(b-2)+\cdots+2}=\delta^{b^{2}-b}, \\
\#\left(\{\text { red spots }\} \cap{ }_{\left[P_{c}\right.} /^{I}[) \leq m \leq b-1 .\right.
\end{array}\right.
$$

The rest of the proposition is assured by Lemma B.2(c).

\section{Appendix C. Definition and properties of $\mathcal{B}, \mathcal{A}$}

A child, of a critical puzzle piece $P$ is a critical puzzle piece $J$, that is a pullback of $P$, such that $]_{J} /^{P[}$ does not meet any critical vertex; see Figure 2 .

Lemma C.1. The hypothesis of recurrence is equivalent to the same condition but with successors replaced by children (this is commonly called persistently recurrent).

Proof. Assume that every critical piece has at most finitely many children. Let $P$ be a critical piece. A successor of $P$ is a grandchild of $P$ of at most $2 b$-th generation. Denote by $n_{0}$ the depth of $P$. Then the depths of the children of $P$ is bounded, say by $n_{1}$. There are finitely many critical pieces of depth $>n_{0}$ and $\leq n_{1}$. So their children all together have a common bound of depths, say $n_{2}$. Hence $n_{2}$ bounds the depth of the grandchildren of $P$. Now the common bound of children's depths of critical pieces of depth $>n_{0}$ and $\leq n_{2}$ bounds also the next generation of grandchildren of $P$, and so on.

Conversely, assume the hypothesis of recurrence. Let $Q$ be a child of a critical piece $P \ni c$, with $c \in C r i t(f)$. Then $\mathcal{L}_{c}(Q)$ exists and is a successor of $P$. As $P$ has only finitely many successors, it has only finitely many children. 
We now come to the constructions of $\mathcal{A}$ and $\mathcal{B}$ :

Proof of Lemma 4.4. We will switch the notation of the red colored critical point from $\mathbf{c}$ to $c_{0}$, and use the letter $c$ to denote an arbitrary critical point. Given a critical puzzle piece $I$ (containing $c_{0}$ ), let $\left\{P_{c} \ni P_{c}^{\prime} \ni c \mid c \in \operatorname{Crit}(f)\right\}$ be the collection of pairs of critical puzzle pieces constructed in Proposition B.1.

Given any $c \in \operatorname{Crit}(f)$ the piece $P_{c}$ has finitely many children. Choose $c_{1}$ and $Q$ such that ${ }_{[Q} /{ }^{P_{c_{1}}[}$ is a longest child-parent diagonal among all children of $P_{c}$ of all $c$.

Set $v=\left.\right|_{Q} /^{P_{c_{1}}} \mid$. Denote by $c^{\prime}$ the critical point contained in $Q$. So $c^{\prime} \in Q$ and $f^{v}(Q)=P_{c_{1}}$.

We have $f^{v}\left(c^{\prime}\right) \in P_{c_{1}}^{\prime}$ (for otherwise apply Proposition B.1 to $x=f^{v}\left(c^{\prime}\right)$ we obtain a child-parent diagonal segment, starting from a piece containing $c^{\prime}$, with size larger than $v$ ). This means that $P_{c_{1}}^{\prime}$ appears on the $v$-th column of $\mathcal{T}\left(c^{\prime}\right)$.

Let $Q^{\prime}$ then be the piece on the 0 -th column of $\mathcal{T}\left(c^{\prime}\right)$ so that $\begin{gathered}Q \\ Q^{\prime}\end{gathered}$ and $\begin{gathered}P_{c_{1}} \\ P_{c_{1}}^{\prime}\end{gathered}$ form a parallelogram. We then obtain our first vacuous segment $\begin{gathered}Q \\ I \\ Q^{\prime}\end{gathered}$, i.e. $\left(Q \backslash \overline{Q^{\prime}}\right) \cap \widehat{\mathcal{P}}_{f}=\emptyset$ : for otherwise let $x \in\left(Q \backslash \overline{Q^{\prime}}\right) \cap \widehat{\mathcal{P}}_{f}$ set $y=f^{v}(x)$. By above $f^{v}$ maps $Q \backslash \overline{Q^{\prime}}$ onto $P_{c_{1}} \backslash \overline{P_{c_{1}}^{\prime}}$. So $f^{v}(y) \in P_{c_{1}} \backslash \overline{P_{c_{1}}^{\prime}}$. Applying now Proposition B.1 to $y$, one again gets a child-parent diagonal segment passing through $x$ and $y$ and with size larger than $v$.

TABLE 1

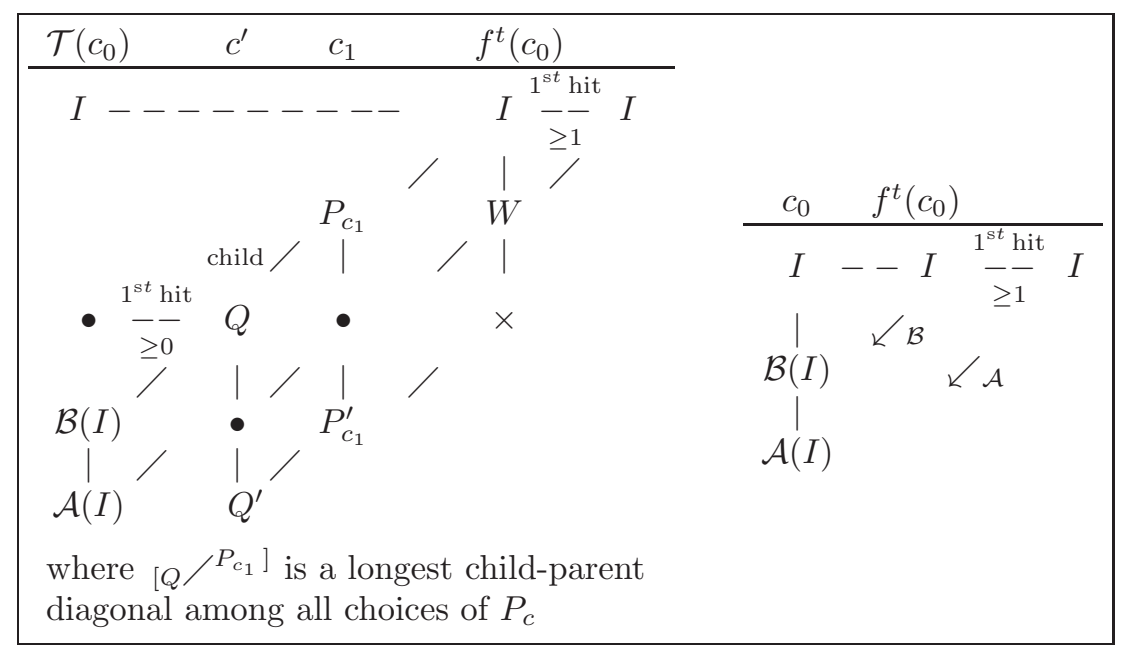

Set now $\mathcal{B}(I)=\widehat{\mathcal{L}}_{c_{0}}(Q)$, the pullback of the first $\geq 0$ hit of $c_{0}$ to $Q$. Then $f^{k}(\mathcal{B}(I))=Q$ for some $k \geq 0$. The fact that $\left(Q \backslash \overline{Q^{\prime}}\right) \cap \widehat{\mathcal{P}}_{f}=\emptyset$ implies $f^{k}\left(c_{0}\right) \in Q^{\prime}$. Therefore the $k$-th column of $\mathcal{T}\left(c_{0}\right)$ contains a vertical segment of critical pieces of $c^{\prime}$ until at least $Q^{\prime}$. Set $t=k+v+l_{c_{1}}$ (where $l_{c_{1}}$ is the integer such that $\left.f^{l_{c_{1}}}\left(P_{c_{1}}\right)=I\right)$, and let $W=\mathcal{L}_{f^{t}\left(c_{0}\right)}(I)$. The fact that $f^{k}\left(c_{0}\right) \in Q^{\prime}$ implies that $f^{t}\left(c_{0}\right) \in f^{l_{c_{1}}}\left(P_{c_{1}}^{\prime}\right)$. Now $f^{l_{c_{1}}}\left(P_{c_{1}}^{\prime}\right)$ is also a pullback of $I$, so $f^{l_{c_{1}}}\left(P_{c_{1}}^{\prime}\right) \subset W$ (as $W$ is the first pullback of $I$ containing $\left.f^{t}\left(c_{0}\right)\right)$. We may then define $\mathcal{A}(I)$ to be 
the pullback by $f^{t}$ of $W$ containing $c_{0}$, i.e. $\mathcal{A}(I), \mathcal{B}(I), I$ and $W$ form a vertical parallelogram in $\mathcal{T}\left(c_{0}\right)$.

Now $\operatorname{deg}\left(\mathcal{B}(I) /^{I}\right) \leq C_{\mathcal{B}}:=\alpha \delta C_{P}=\delta^{b} C_{P}$, and $\operatorname{deg}\left(\mathcal{A}(I)^{I}\right) \leq C_{\mathcal{A}}:=\delta^{b} C_{B}=$ $\delta^{2 b} C_{P}$.

The control of numbers of red spots follows from the control for $P_{c_{1}}$ and the fact that $\left.{ }_{\mathcal{B}(I)} /{ }^{P_{c_{1}}[\text { and }}\right] W{ }^{I[}$ have no red spots, and the piece $W$ may or may not be red.

By Rule 3, $\begin{gathered}Q \\ Q^{\prime}\end{gathered}$ vacuous implies that $\begin{gathered}\mathcal{B}(I) \\ \mathcal{A}(I)\end{gathered}$ is vacuous.

\section{Appendix D. Case there is only one critical point $(b=1)$}

Assume now that $f$ has only one critical point, denoted by $c$. The above proof works for $\tau \geq 2$. We show here how to make it work for $\tau=1$.

Now the notion of a child coincides with that of a successor. Thus Lemma 4.2 claims that any critical piece has at least two and at most finitely many children (see TY], Lemma 1.3 for a simpler proof adapted to this situation). As $\tau=b=1$, we have $\Gamma^{\tau}=\Gamma$, i.e. $\Gamma^{\tau}$ is simply the last child operator.

Corollary 4.3 is still valid, with the same proof. The operators $\mathcal{B}$ and $\mathcal{A}$ are now defined as

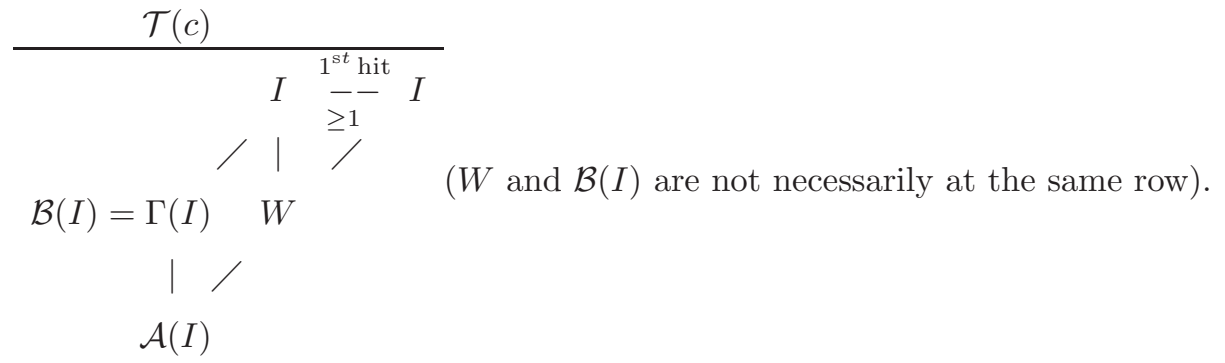

This is consistent with Table 1 , by taking $P_{c_{1}}=I, P_{c_{1}}^{\prime}=W, Q=\mathcal{B}(I)$ and $Q^{\prime}=\mathcal{A}(I)$.

New proof of Lemma 4.4. (the study of Appendix B is no longer needed).

At first $W$ has to be critical (for otherwise $\mathcal{A}(I)$ is a child of $I$ deeper than $\left.\Gamma^{\tau}(I)\right)$. Apply Rule 2 to the two triangles, one with vertices $W, I, I$, the other with vertices $\mathcal{L}_{c}(I), I, I$, one sees that $W=\mathcal{L}_{c}(I)$.

Now assume by contradiction that $f^{t}(c) \in \mathcal{B}(I) \backslash \overline{\mathcal{A}(I)}$ for some $t>0$. This is seen in $\mathcal{T}(c)$ as below, with $E$ noncritical:

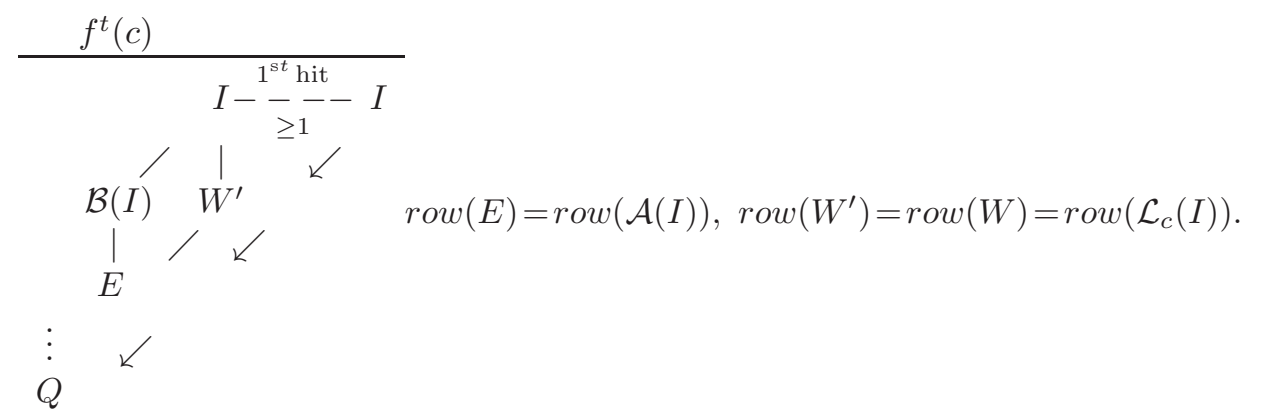


Consider the two parallelograms, the first with vertical edges $\begin{gathered}\mathcal{B}(I) \\ \begin{array}{l}\mathcal{A}(I) \\ \end{array}\end{gathered}$ and $\begin{gathered}I \\ I\end{gathered}$, the

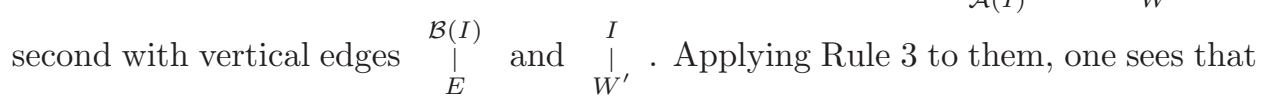
$W^{\prime}$ is not critical. Now start from the upper-right vertex of the second parallelogram and march right until the first critical vertex, and from there march diagonally leftdownwards until the first critical vertex $Q$. Now $Q$ cannot be on $\operatorname{column}\left(W^{\prime}\right)$ (for otherwise $Q$ has to be above $W^{\prime}$ and one gets a smaller triangle than the one with vertices $\left.I, I, \mathcal{L}_{c}(I)\right)$. Then $Q$ must be a child of $I$ deeper than $\mathcal{B}(I)=\Gamma(I)$. This is not possible.

Therefore $\begin{gathered}\mathcal{B}(I) \\ \mathcal{A}(I)\end{gathered}$ is vacuous. (This is actually the major simplification compared with the case $b>1$.)

One may now construct the KSS nest $\left(K_{n}^{\prime}, K_{n}\right)_{n}$ from a critical piece $K_{0}$ exactly as in Figure 5, i.e. $K_{n}^{\prime}=\mathcal{B} \Gamma\left(K_{n-1}\right)=\Gamma^{2}\left(K_{n-1}\right)$ and $K_{n}=\mathcal{A} \Gamma\left(K_{n-1}\right)$ (this is also consistent with the construction in [TY], Figure 2.2).

Now for any $m \geq 1$ make the same construction as in Figure 3 (this is slightly better than the construction in [TY, as $m+4$ there has been replaced by $m+2$ here). It remains to prove Proposition 3.2

As $\tau=b=1$, we will have to sharpen the estimates in Lemma 4.5, We keep the same notation.

By Corollary 4.3(iii), we have $2 r(J) \leq\left.\right|_{\mathcal{B}(J)} /^{J}|=|_{\Gamma(J)} /^{J} \mid \leq r(\mathcal{B}(J))$, therefore $3 r(J) \leq\left.\right|_{\mathcal{A}(J)} /^{J} \mid \leq 2 r(\mathcal{A}(J))$, by the new construction of $\mathcal{A}$ and Corollary 4.3(ii). This implies successively the following estimates (replacing Lemma 4.5 and (18)):

$$
\begin{gathered}
3 r\left(I_{n}\right) \leq u_{n} \leq 2 r\left(K_{n}\right) \leq q_{n}=\left.\right|_{I_{n+1}=\Gamma\left(K_{n}\right)} /^{K_{n}} \mid \leq r\left(I_{n+1}\right) \\
r\left(I_{n}\right) \leq \frac{1}{3} u_{n} \leq \frac{2}{3} r\left(K_{n}\right), \quad p_{n}=q_{n-1}+u_{n} \leq r\left(I_{n}\right)+u_{n} \leq \frac{4}{3} u_{n} \leq \frac{8}{3} r\left(K_{n}\right) ; \\
p_{n+1}=q_{n}+u_{n+1} \geq r\left(K_{n}\right)+3 r\left(I_{n+1}\right) \geq r\left(K_{n}\right)+3 \cdot 2 r\left(K_{n}\right)=8 r\left(K_{n}\right) \geq 3 p_{n} \\
\left.\right|_{K_{n}} /^{K_{0}} \mid=p_{n}+\cdots+p_{1}<\left(1+\frac{1}{3}+\frac{1}{3^{2}}+\cdots\right) p_{n}=\frac{3}{2} p_{n} .
\end{gathered}
$$

New proof of Proposition 3.2. Points (a), (b) and (d) are proved as in 4.5 . Formula (19) becomes now: $(L-1) u_{m} \leq M<\frac{3}{2} p_{m} \leq \frac{3}{2} \cdot \frac{4}{3} u_{m}=2 u_{m}$. So $L \leq 2$. Point (c) follows as in 4.5 with $d=\left(C_{\mathcal{A}} \alpha\right)^{2}$. To get Point (e) we replace (20) and (21) by (the critical pieces are colored red):

$$
\begin{aligned}
& \text { \#\{red-spots } \in \operatorname{top}(T)\}-1 \stackrel{\text { def. of } L}{\leq} L \cdot(b+1) \leq 2(b+1)=4 \\
& \left|\Gamma\left(K_{m}\right) /^{K_{m}}\right| \cdot\left(\#\left\{\text { red-spots } \in \operatorname{top}\left(T^{\prime}\right)\right\}-1\right) \\
& \stackrel{\text { Corollary } 4.3 \text { iii) }}{\text { ii }} R\left(K_{m}\right) \cdot\left(\#\left\{\text { red-spots } \in \operatorname{top}\left(T^{\prime}\right)\right\}-1\right) \\
& \stackrel{\text { as in } 211}{\geq} \quad r\left(I_{m+2}\right)=\left.\frac{r\left(I_{m+2}\right)}{r\left(I_{m+1}\right)} r\left(I_{m+1}\right) \stackrel{29}{\geq} 3 \cdot\right|_{\Gamma\left(K_{m}\right)} / K_{m} \mid .
\end{aligned}
$$

It follows then $\#\left\{\right.$ red-spots $\left.\in \operatorname{top}\left(T^{\prime}\right)\right\}-1 \geq 4 \geq \#\{$ red-spots $\in \operatorname{top}(T)\}-1$. Point (e) follows. 
We sketch now a construction in the spirit of Branner-Hubbard, for the sake of comparison.

Fix a choice of a critical puzzle piece $I$. We choose $r=\left|\begin{array}{c}I \\ \mid \\ \mathcal{L}_{c}(I)\end{array}\right|$ (or simply $r=1$ as in $[\mathrm{BH}]$ ). For any critical puzzle piece $J$ define $\mathcal{D}(J)$ to be the puzzle piece in the same critical nest by adding $r$ depths.

Set $I_{0}=\mathcal{D}^{-1} \mathcal{L}_{c} \mathcal{D}(I), I_{1}=\Gamma(I)$. For any finite words $w$ in the alphabet $\{0,1\}$, set inductively $I_{0 w}=\mathcal{D}^{-1} \mathcal{L}_{c} \mathcal{D}\left(I_{w}\right)$ and $I_{1 w}=\Gamma\left(I_{w}\right)$.

We claim then $\left\{I_{w} \backslash \overline{\mathcal{D}\left(I_{w}\right)}, w\right\}$ are pairwise disjoint, each is a pullback of $I \backslash \overline{\mathcal{D}(I)}$ and takes $1 / \delta^{|w|}$ portion of the modulus.

At first we show that each of $\begin{gathered}I_{0} \\ 1 \\ \mathcal{D}\left(I_{0}\right)\end{gathered}$ and $\begin{gathered}I_{1} \\ 1 \\ \mathcal{D}\left(I_{1}\right)\end{gathered}$ forms a parallelogram with $I$
$\mathcal{D}(I)$ whose upper diagonal edge does not contain critical vertices (except the two ending vertices). For the first parallelogram this follows from Rules 1 and 2, and for the second it follows from what we have just proved above about the pair $(\Gamma(I), \mathcal{A}(I))$.

Next we prove $\mathcal{D}\left(I_{0}\right) \supsetneq I_{1}$ as in M1]: Let $t=\left|\begin{array}{l}I \\ I_{1}\end{array}\right|$. Denote by $E$ the last critical entry of the $t$-th column of $\mathcal{T}(c)$, and by $Q$ the vertex on $\operatorname{row}(I)$ forming a diagonal segment with $E$. Now go to the first critical vertex strictly on the right of $Q$ and then follow the left-down diagonal there all the way to the 0 -th column. Denote that vertex by $S$. Applying Rule 3 repeatedly, one could prove that $S$ is a child of $I$ (for details, see [M1]). Consequently $\mathcal{D}\left(I_{0}\right) \supsetneq S \supset \Gamma(I)=I_{1}$.

We omit the rest of the details. In case $\delta=2$ (simple critical point), no modulus is lost from words of length $n$ to those of length $n+1$. Now Grötzsch's inequality alone, without the Kahn-Lyubich covering lemma, is enough to guarantee that the diameter of the critical puzzle pieces shrinks to zero.

\section{TABLE OF CONSTANTS}

$b=\#$ Crit $(f), \quad \delta:=\sup _{c \in \operatorname{Crit}(f)} \operatorname{deg}_{c}(f)$. The following constants depend only on $b$ and $\delta: \alpha=\delta^{b-1}, \quad C_{\Gamma}=\delta^{2 b-1}, \quad C_{\mathcal{B}}=\delta^{b^{2}}, \quad C_{\mathcal{A}}=\delta^{b^{2}+b}$.

For the short-cutted KSS nest: $\tau=b, C_{K}=C_{\mathcal{A}} C_{\Gamma}^{\tau}, \beta=C_{\mathcal{B}} C_{\Gamma}^{\tau} C_{K}, d=\left(C_{\mathcal{A}} \alpha\right)^{4}$.

For the original KSS nest: $\tau=b+2, C_{K}=C_{\mathcal{B}} C_{\mathcal{A}} C_{\Gamma}^{\tau}, \beta=C_{\mathcal{B}}^{2} C_{\Gamma}^{\tau} C_{K}, d=$ $\left(C_{\mathcal{B}} \alpha\right)^{2(b+4)}$.

$Z=2 d^{3} \alpha^{2} \beta^{2}+1, m \geq Z, \eta=\frac{1}{d \alpha \beta}, D=C_{K}^{Z}, \varepsilon(\eta, D)$ is the Kahn-Lyubich constant, $C(b, \delta, \widehat{\mu})=\min \left\{\frac{1}{\beta} \cdot \varepsilon(\eta, D), \frac{1}{C_{K}^{Z+1}} \widehat{\mu}\right\}$.

\section{ACKNOWLEDGEMENTS}

The authors would like to thank Shen Weixiao for his deep insight and influence to this work and also thank the referees for carefully reading the paper. The first and fourth authors also want to thank Cergy-Pontoise University where most of this work has been done. 


\section{REFERENCES}

[AKLS] A. Avila, J. Kahn, M. Lyubich and W. Shen, Combinatorial rigidity for unicritical polynomials, ArXiv: math.DS/0507240. Annals of Math, Sept. 2009.

[BH] B. Branner and J. H. Hubbard, The iteration of cubic polynomials, Part II: Patterns and parapatterns, Acta Math., 169 (1992), 229-325. MR.1194004(94d:30044)

[KL1] J. Kahn and M. Lyubich, The quasi-additivity law in conformal geometry, Annals of Math. 169 (2009), No. 2, 561-593. MR2480612

[KL2] J. Kahn and M. Lyubich, Local connectivity of Julia sets for unicritical polynomials, ArXiv: math.DS/0505194, Stony Brook IMS preprint 2005/3. Annals of Math, Sept. 2009.

[KSS] O. Kozlovski, W. Shen, and S. van Strien, Rigidity for real polynomials, Annals of Mathematics 165 (2007), 749-841. MR2335796 (2008m:37063)

[KS] O. Kozlovski and S. van Strien, Local connectivity and quasi-conformal rigidity of nonrenormalizable polynomials, ArXiv: math.DS/0609710. Accepted for publication in the Proceedings of the LMS.

[M1] J. Milnor, Local connectivity of Julia sets: expository lectures. In: The Mandelbrot set, Theme and Variations, edited by Tan Lei, London Math. Soc. Lecture Note Ser., No 274, Cambridge Univ. Press, 2000, 67-116. MR:1765085 (2001b:37073)

[M2] John Milnor, Dynamics in One Variable, Annals of Mathematics Studies, 160, Princeton University, 2006. MR2193309 (2006g:37070)

[QY] Qiu Weiyuan and Yin Yongcheng, Proof of the Branner-Hubbard conjecture on Cantor Julia sets, Science in China, Series A, 2009, Vol. 52, No. 1, 45-65. MR2471515 (2009j:37074)

[PT] Peng Wenjuan and Tan Lei, Combinatorial rigidity of unicritical maps, to appear in: Science in China.

[Ro] Pascale Roesch, Puzzles de Yoccoz pour les applications à allure rationnelle, L'Enseignement Mathématique, tome 45, Juin 1999, pp. 133-168. MR 1703365 (2000g:37050)

[RY] Pascale Roesch and Yin Yongcheng, The boundary of bounded polynomial Fatou components, C. R. Acad. Sci. Paris, Ser. I 346 (2008). MR2441925

[TY] Tan Lei and Yin Yongcheng, Unicritical Branner-Hubbard conjecture, in Family and friends, ed. Dierk Schleicher.

[YZ] Yin Yongcheng and Zhai Yu, No invariant line fields on Cantor Julia sets, to appear in: Forum Mathematicum.

School of Mathematical Sciences, Peking University, Beijing, 100871, People's RePUBLIC OF CHINA

E-mail address: wenjpeng@amss.ac.cn

School of Mathematical Sciences, Fudan University, Shanghai, 200433, People's RePUBLIC OF CHINA

E-mail address: wyqiu@fudan.edu.cn

Laboratoire Émile-Picard, Université Paul-Sabatier, 118, route de Narbonne, 31062 Toulouse Cedex 9, France

E-mail address: roesch@math.univ-toulouse.fr

Université D'Angers, Faculté des Sciences, LAREMA, 2, Boulevard Lavoisier, 49045 Angers Cedex 01, France

E-mail address: Lei.Tan@univ-angers.fr

School of Mathematical Sciences, Fudan University, Shanghai, 200433, People's RePUBLIC OF CHINA

E-mail address: ycyin@fudan.edu.cn 